\title{
Late Holocene uplift of the Izu Islands on the northern Zenisu Ridge off Central Japan
}

\author{
Akihisa Kitamura ${ }^{1,2^{*}}$, Takafumi Imai ${ }^{1}$, Yuta Mitsui ${ }^{1,2}$, Mami Ito ${ }^{1}$, Yosuke Miyairi ${ }^{3}$, Yusuke Yokoyama ${ }^{3}$ \\ and Yuki Tokuda ${ }^{4}$
}

\begin{abstract}
To clarify the Holocene uplift history of the Izu Islands, Japan, we analyze the elevations and ${ }^{14} \mathrm{C}$ ages of emerged sessile assemblages measured by accelerator mass spectrometry (AMS) on the islands of Niijima, Jinaijima, Shikinejima, and Kouzushima, on the northern Zenisu Ridge. The results suggest that uplift events took place after AD 1950 (uplift event 1), during AD 786-1891 (uplift event 2), during AD 600-1165 (uplift event 3), and during AD 161-686 (uplift event 4), although uplift events 3 and 4 are identified only at Kouzushima. The minimum amount of uplift was estimated to be $0.4-0.9 \mathrm{~m}$ in uplift event 1, 2.4-2.7 $\mathrm{m}$ in uplift event 2, 3.6 m in uplift event 3, and 3.3-8.1 $\mathrm{m}$ in uplift event 4 . These events could have been caused by volcanic activity or strong earthquakes. There also remains the possibility that uplift event 2 was caused by the AD 1498 Meio earthquake; in contrast to the previous interpretation, the ages of uplift events are significantly older than the earthquake, based on conventional (non-AMS) methods.
\end{abstract}

Keywords: Emerged marine sessile assemblage, ${ }^{14} \mathrm{C}$ date, Holocene, Niijima, Shikinejima, Kouzushima

\section{Introduction}

Fukutomi $(1935,1938)$ discovered emerged sessile assemblages on Shikinejima and Jinaijima within the Izu Islands and in southern Izu Peninsula, Central Japan (Figs. 1 and 2). He suggested that these assemblages provided evidence of coseismic uplift associated with the $A D$ 1703 great Kanto (Genroku) earthquake (Fig. 1), although the ages of the assemblages were not obtained. On the other hand, Hatori (1975) and Aida (1981) interpreted the same assemblages as evidence of the AD 1498 Meio earthquake (M 8.2-8.4) (Fig. 1). These authors suggested that the tsunami believed to have been associated with the Meio earthquake affected coastal regions from Kii Peninsula to Boso Peninsula, Central Japan (Fig. 1b) and hypothesized that the rupture area included the western and eastern Tōnankai segments $(\mathrm{C}$ and $\mathrm{D}$ in Fig. 1a, respectively) and the area south of Izu Peninsula.

\footnotetext{
*Correspondence: kitamura.akihisa@shizuoka.ac.jp

${ }^{1}$ Institute of Geosciences, Shizuoka University, 836 Oya, Suruga-ku, Shizuoka 422-8529, Japan

${ }^{2}$ Center for Integrated Research and Education of Natural Hazards, Shizuoka University, 836 Oya, Suruga-ku, Shizuoka 422-8529, Japan

Full list of author information is available at the end of the article
}

Ota et al. $(1983,1986)$ determined the ${ }^{14} \mathrm{C}$ ages of emerged assemblages from the southern coast of Izu Peninsula and Shikinejima (Fig. 2; Table 1), and demonstrated that the ages obtained were inconsistent with the hypotheses of Fukutomi $(1935,1938)$, Hatori (1975), and Aida (1981). However, these ages were not calibrated. Kitamura et al. (2016) examined the faunal compositions and accelerator mass spectrometry (AMS) ${ }^{14} \mathrm{C}$ ages of emerged sessile assemblages in the southern part of Izu Peninsula and concluded that coseismic uplift occurred in the periods 1256950 BC, 1000-1270 AD, 1430-1660 AD, and 15061815 AD. Kitamura et al. (2015) found no evidence of Holocene coseismic uplift at Mikomotojima, a small island located $10 \mathrm{~km}$ off the coast of Shimoda and inferred that the source fault for the uplift events was a reverse fault located $\sim 3 \mathrm{~km}$ offshore from southern Izu Peninsula (Fig. 1c). On the other hand, AMS ${ }^{14} \mathrm{C}$ ages have yet to be determined for the emerged sessile assemblages on Shikinejima. In addition, we know of no published studies of emerged sessile assemblages on Niijima or Kouzushima. Thus, the present study examines Holocene crustal movements on the islands of Shikinejima, Niijima, and 


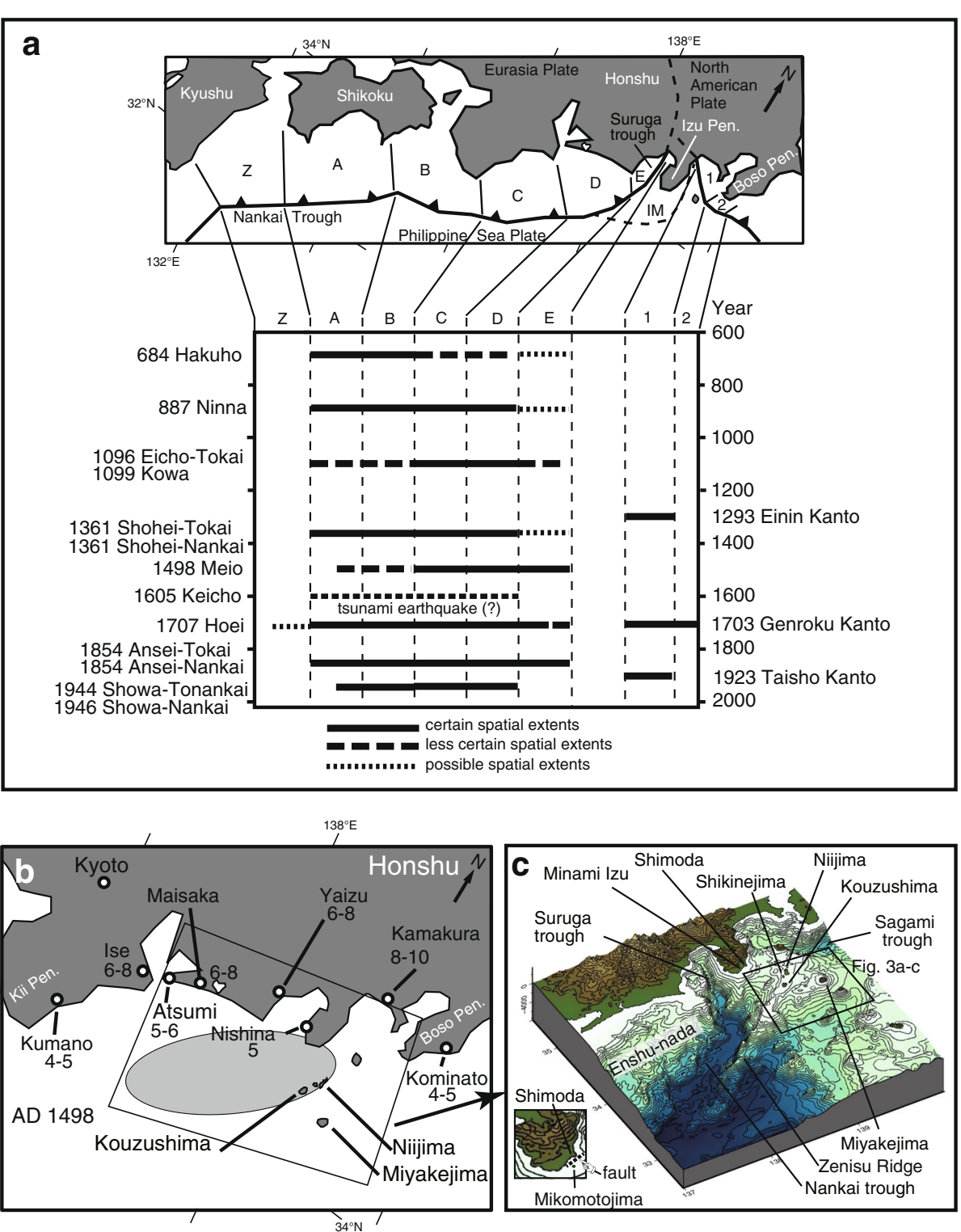

Fig. 1 a Spatio-temporal distribution of great earthquakes along the Nankai, Suruga, and Sagami troughs (Ando 1975; Ishibashi and Satake 1998; Shishikura 2014). Individual fault segments are labeled A-E and $Z_{;} 1$ indicates the Sagami Bay segment and 2 denotes the South Boso segment. The boundary of the Izu microplate (IM) is after Nishimura et al. (2007). b Distribution of inundation heights for the Meio earthquake, as determined by Hatori (1975). The hatched area indicates the source area of the Meio earthquake (Hatori 1975). c Map of the study area and Zenisu Ridge. The submarine fault between Shimoda and Mikomotojima is after Kitamura et al. (2015)

Kouzushima, using elevation data and AMS ${ }^{14} \mathrm{C}$ dating of emerged marine sessile assemblages.

\section{Study area}

Niijima, Jinaijima, Shikinejima, and Kouzushima are located along the northern part of the Zenisu Ridge on the northern Philippine Sea Plate (Fig. 1). The Zenisu Ridge is a topographic high composed of oceanic crust (Le Pichon et al. 1987; Chamot-Rooke and Le Pichon 1989; Ishihara 1989; Lallemant et al.
1989; Nakanishi et al. 1994; Fig. 1). Using regional kinematic models, Mazzotti et al. $(1999,2001)$ examined the movement of crustal blocks around Central Japan and identified three blocks: the Philippine Sea Plate, Central Japan, and the Zenisu-West Izu block. Sagiya (1999) and Nishimura et al. (2007) refer to the Zenisu-West Izu block as the Izu microplate (Fig. 1). A subduction thrust occurs offshore from the Tokai area along the southern edge of the microplate (Nakanishi et al. 1994). The eastern boundary of the 


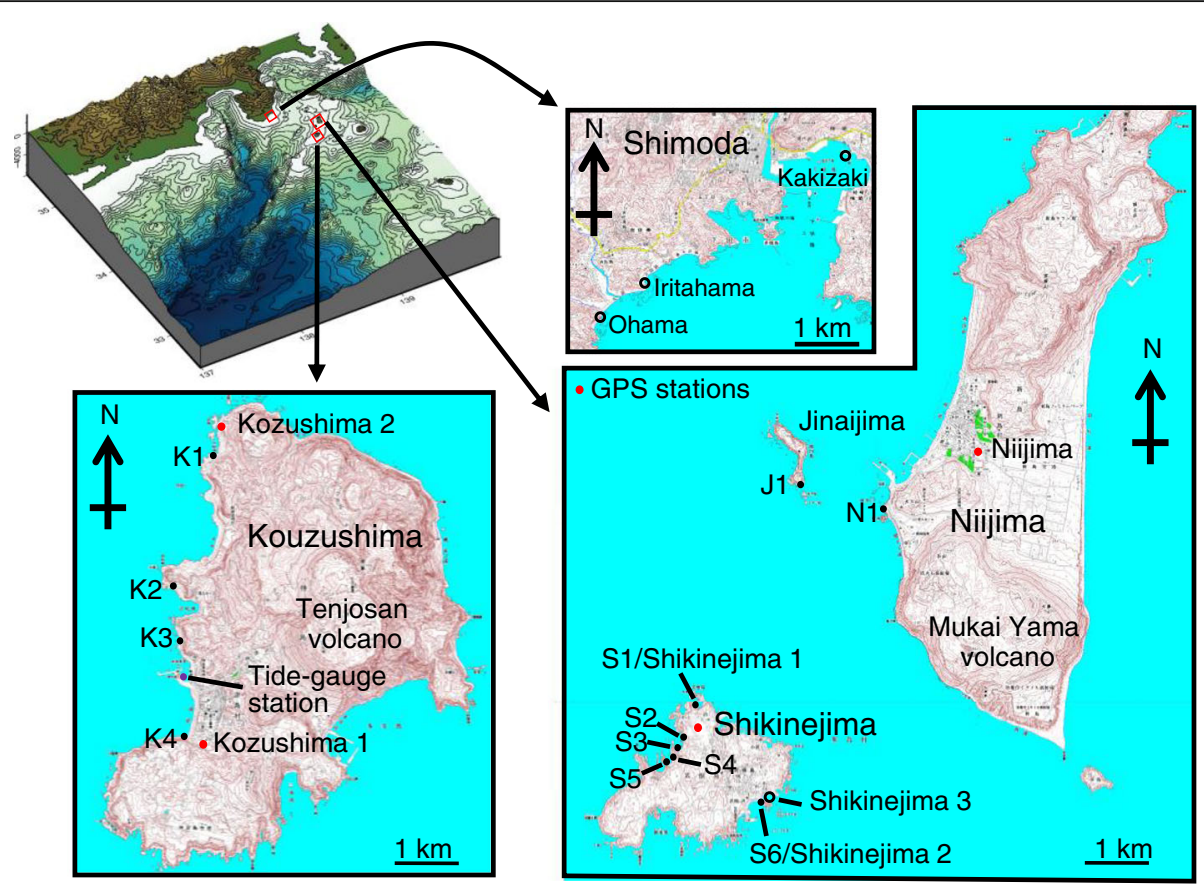

Fig. 2 Locality map of field sites on Shimoda, Niijima, Jinaijima, Shikinejima, and Kouzushima, showing sampling sites investigated by Ota et al. (1983, 1986). The base map is from the 1:200,000 topographic maps "Shimoda," "Mikomojima," "Niijima," and "Kouzushima" published by the Geospatial Authority of Japan. Red dots indicate GPS stations

microplate consists of a sinistral strike-slip fault zone (Mazzotti et al. 1999, 2001) that is located less than $10 \mathrm{~km}$ west of Shikinejima (Nishimura et al. 2007).

Niijima, Jinaijima, Shikinejima, and Kouzushima consist mainly of Quaternary volcanic rocks (Isshiki 1987). Historical documents reveal that Mukaiyama volcano on Niijima and Tenjosan volcano on Kouzushima (Fig. 2) erupted in $\mathrm{AD} 886$ and $\mathrm{AD}$ 838, respectively (Isshiki

Table $1{ }^{14} \mathrm{C}$ dates reported by Ota et al. $(1983,1986)$. Area and sampling points are shown in Fig. 2

\begin{tabular}{llll}
\hline $\begin{array}{l}\text { Area/sampling } \\
\text { points }\end{array}$ & Materials & $\begin{array}{l}{ }^{14} \text { C age } \\
\text { (years BP) }\end{array}$ & $\begin{array}{l}\text { Sample height } \\
(\mathrm{m})\end{array}$ \\
\hline Ohama & Chthamalus sp. & $2650 \pm 80$ & $2.3-2.7$ \\
Ohama & Saccostrea echinata & $2830 \pm 90$ & $2.3-2.7$ \\
Ohama & Saccostrea echinata & $670 \pm 75$ & 1.1 \\
Ohama & Saccostrea echinata & $645 \pm 80$ & 0.9 \\
Iritahama & Tetraclitella chinensi & $950 \pm 90$ & $1.45-2.15$ \\
Kakizaki & Saccostrea echinata & $1280 \pm 75$ & 1.6 \\
Kakizaki & Saccostrea echinata & $1540 \pm 65$ & 1.6 \\
Shikinejima 1 & Tetraclitella chinensi & $1980 \pm 130$ & $3.2-3.6$ \\
Shikinejima 1 & Megabalanus rosa & $1830 \pm 130$ & $3.0-3.1$ \\
Shikinejima 1 & Tetraclitella chinensi & $1350 \pm 100$ & 2.6 \\
Shikinejima 1 & Saccostrea kegaki & $2540 \pm 290$ & 2.6 \\
Shikinejima 2 & Tetraclita japonica & $1010 \pm 300$ & 4.2 \\
Shikinejima 3 & Tetraclitella chinensi & $1530 \pm 100$ & $3.9-5.1$ \\
\hline
\end{tabular}

1987). Togashi (1984) suggested that volcanic activity also occurred about 230 years before the AD 838 Kouzushima eruption.

Earthquakes in AD 1890 (M 6.8) and 1936 (M 6.3) damaged Shikinejima and Niijima, respectively (Hagiwara and Omote 1936; Honda 1938; Hamada 2001), with epicenters located approximately $14 \mathrm{~km}$ and $1 \mathrm{~km}$ south of Niijima Island, respectively (Hamada 2001; Fig. 3). The focal mechanisms of both earthquakes (Honda 1938) indicate strike-slip motion (Fig. 3d).

Recent crustal movements in the study area have been inferred from tide gauge and Global Positioning System (GPS) data. Tide-gauge data indicate about 0.9 m of uplift of Kouzushima Island between 1990 and 2014 (Coastal Movements Data Center, 2016; Fig. 4a). GPS time-series data of the F3 solution (Nakagawa et al. 2009) of GEONET, provided by the Geospatial Information Authority of Japan, reveal that Shikinejima and Niijima are being uplifted at a mean rate of $1.0 \mathrm{~cm} /$ year since 1998 (Fig. 4b). This uplift is related to a pressure source located beneath northeastern Kouzushima (Kimata et al. 2000) and dike intrusion associated with the AD 2000 eruption of Miyakejima volcano, southeast of Kouzushima (Fig. 2) (Murakami et al. 2001; Nishimura et al. 2001; Yamaoka et al. 2005).

The coastlines in the study area are characterized by a wave-dominated and microtidal regime, with a maximum tidal range of $1.6 \mathrm{~m}$ during spring tides. In this 
a

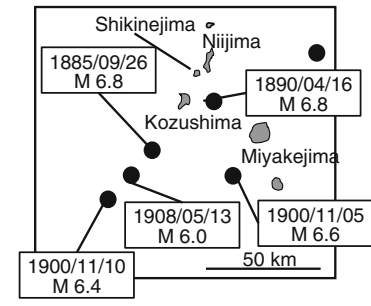

1 January 1885 to 31 December 1925

C

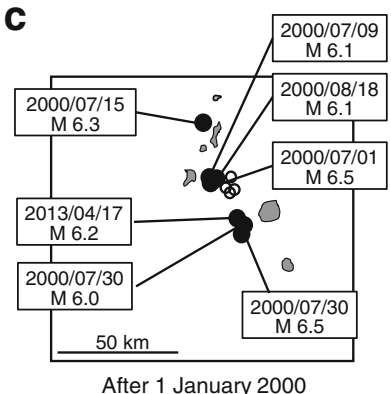

b

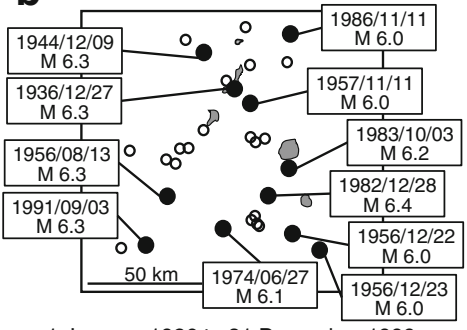

1 January 1926 to 31 December 1999

d

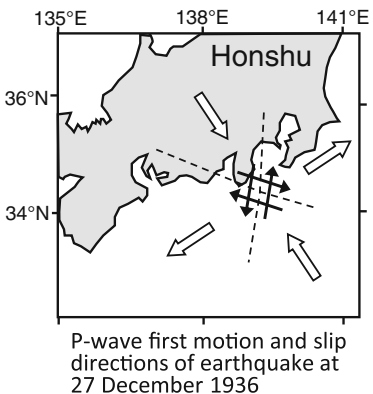

Fig. 3 a, b Epicentral locations of significant earthquakes in the study area (Hamada 2001). c Epicentral locations of significant earthquakes in the study area based on the hypocenter catalog of the Japan Meteorological Agency. d P wave first motions of the 27 December 1936 earthquake (Honda, 1938) with possible slip directions indicated by black arrows

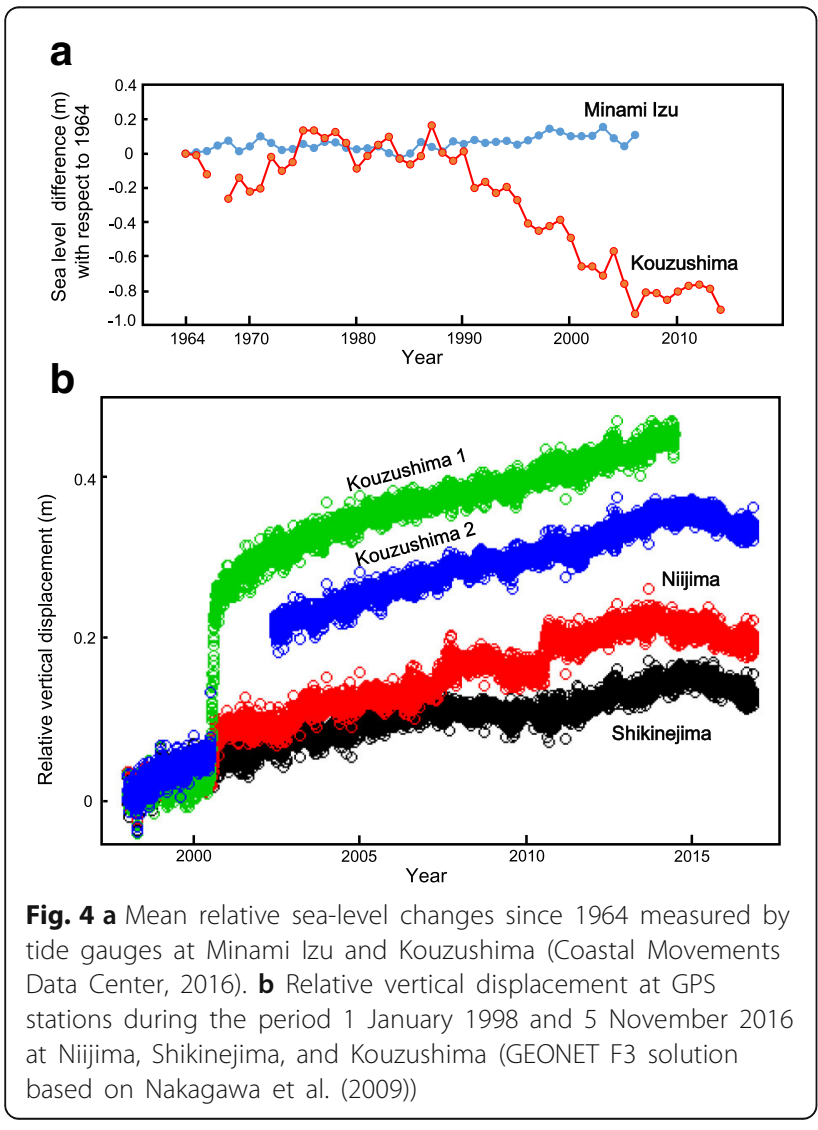

study, the intertidal zone ranges between -0.8 and $0.8 \mathrm{~m}$ above mean sea level (amsl).

\section{Methods/Experimental}

This study examines emerged marine sessile assemblages at one site on Niijima, one site on Jinaijima, six sites on Shikinejima, and four sites on Kouzushima (Fig. 2). At each site, we collected several samples of well-preserved barnacles, bivalves, Serpulidae, and corals. Before the specimens were identified, each was cleaned with a micro-knife and organic matter was removed using hydrochloric acid. The coral samples were analyzed by X-ray photography and X-ray diffraction. Radiocarbon dating was performed by accelerator mass spectrometry (AMS) at the University of Tokyo, Japan (Yokoyama et al. 2016). Radiocarbon ages were then translated into calendar time scales using the program OxCal 4.3 (Bronk Ramsey 1995; Bronk Ramsey and Lee 2013), based on comparisons with Marine 13 data (Reimer et al. 2013). A local correction of $\Delta R=109 \pm 60$ calculated for Shimoda, $42 \mathrm{~km}$ northwest of Shikinejima (Yoneda et al. 2000), was applied to all data. We also observed the upper limit of living hermatypic coral, Saccostrea kegaki and Crassostrea gigas oysters, Tetraclitella chinensis barnacles, and Pomatoleios kraussii tubeworms where we collected the emerged specimens. Since these species occur in high densities, reliable data were easily obtained. 


\section{Results}

\section{Site descriptions and ${ }^{14} \mathrm{C}$ dating}

\section{Niijima}

At site N1, we collected in situ specimens of abraded Saccostrea kegaki at 0.0-0.7 $\mathrm{m}$ amsl and in situ samples of fresh Crassostrea gigas at 0.3-0.9 m amsl (Figs. 2 and 5). We observed the burial of these specimens by sand on 22 September 2014. Although two specimens of $S$. kegaki fall within the age range of AD 1816 to present, all other samples represent modern specimens (Table 2). The upper limit of living $S$. kegaki is $+0.4 \mathrm{~m}$ amsl, while living specimens of $C$. gigas could not be found.

\section{Jinaijima}

Jinaijima is a small island located $2 \mathrm{~km}$ west of Niijima Island (Fig. 2). We collected encrusting masses of Pomatoleios kraussii tubeworms at $0.6 \mathrm{~m}$ amsl at site J1, which is located on bedrock on the east side of the Island (Figs. 2 and 6). The encrusting masses are up to $4 \mathrm{~cm}$ thick and lack any observable layered structure. ${ }^{14} \mathrm{C}$ dating of the surface (J11) and innermost part (J12) of the masses yields ranges of AD 1724 to present and AD 1727 to present, respectively (Table 2). Encrusting masses of living P. kraussii could not be found.

\section{Shikinejima}

Site S1 is a cave located in a cliff south of Tomari Port (Figs. 2 and 7a). The cave is $1 \mathrm{~m}$ wide, $10 \mathrm{~m}$ high, and $4 \mathrm{~m}$ long. Inside the cave, we collected many well-preserved individual samples of the barnacle Tetraclitella chinensis (Fig. 7b) and in situ samples of the bivalve Cardita leana at elevations of $2.6-4.0 \mathrm{~m}$ amsl (Fig. $7 \mathrm{c}$ ). ${ }^{14} \mathrm{C}$ dates of all specimens range from AD 1006 to 1891 (Table 2). Ota et al. (1983) previously collected uplifted sessile assemblages from this site and obtained non-calibrated ${ }^{14} \mathrm{C}$ ages of $1980 \pm 130$ years BP and $1350 \pm 100$ years BP for specimens found at elevations of 3.2-3.6 and $2.6 \mathrm{~m}$ amsl, respectively (Table 1 ). We observed that the upper limit of living $T$. chinensis is about $+0.8 \mathrm{~m}$ amsl.

Site S2 is located on bedrock on the east side of Oura Bay, at about $+3.0 \mathrm{~m}$ amsl (Fig. 2). The ${ }^{14} \mathrm{C}$ age of wellpreserved C. leana is AD 988-1268 (Table 2).

Site S3 is located on bedrock on the west side of Oura Bay, at about $+2.3 \mathrm{~m}$ amsl (Fig. 2). This site yielded many well-preserved examples of $T$. chinensis that yielded ${ }^{14} \mathrm{C}$ dates in the range AD 1216-1505.

Site S4 is located on bedrock on the east side of Nakanoura Bay, at approximately $-0.1 \mathrm{~m}$ amsl (Figs. 2 and $8 \mathrm{a}$ ). This site yielded three in situ colonial zooxanthellate scleractinian corals of Cyphastrea serailia (Fig. 8b), which were $100 \%$ aragonite and therefore not susceptible to diagenesis.
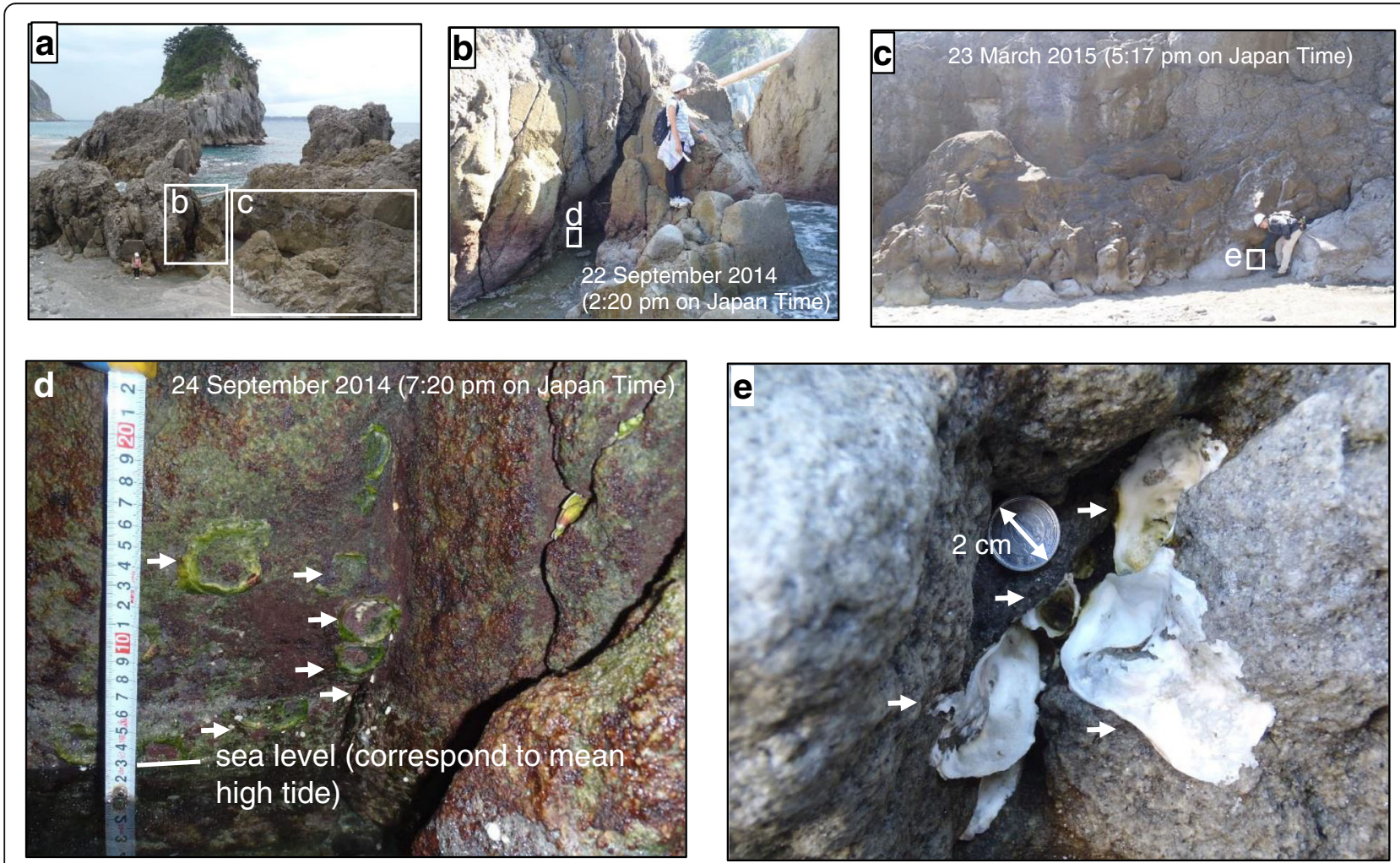

Fig. 5 Photographs of $\mathbf{a}$ the study area at site N1 in Niijima, $\mathbf{b}$ the sampling point at which S. kegaki was collected, $\mathbf{c}$ the sampling point at which C. gigas was collected, d S. kegaki. (arrows indicate individual examples), and e C. gigas (arrows indicate individual examples) 
Table 2 Results of ${ }^{14} \mathrm{C}$ dating in the present study

\begin{tabular}{|c|c|c|c|c|c|c|c|c|c|c|c|}
\hline $\begin{array}{l}\text { Sample } \\
\text { name }\end{array}$ & Locality & Site & $\begin{array}{l}\text { Laboratory } \\
\text { number } \\
\text { (YAUT-) }\end{array}$ & $\begin{array}{l}\text { Coordinates } \\
\text { (lat/long) }\end{array}$ & $\begin{array}{l}\text { Height } \\
(\mathrm{m})\end{array}$ & $\begin{array}{l}\text { Upper- } \\
\text { growth } \\
\text { limit (m) }\end{array}$ & Materials & $\begin{array}{l}\text { Conventional } \\
{ }^{14} \mathrm{C} \text { age (years } \\
\mathrm{BP})\end{array}$ & $\begin{array}{l}\text { Calibrated } \\
\text { age }(2 \sigma)\end{array}$ & Groups & $\begin{array}{l}\text { Total } \\
\text { minimum } \\
\text { uplift (m) }\end{array}$ \\
\hline N1 & Niijima & N1 & 14905 & $\begin{array}{l}34.21499^{\circ} \\
139.14331^{\circ}\end{array}$ & 0.7 & $0.4(0.8)$ & $\begin{array}{l}\text { Saccostrea } \\
\text { kegaki }\end{array}$ & $407 \pm 32$ & $\begin{array}{l}\text { Cal AD } \\
1816- \\
1950\end{array}$ & A & $0.3(-0.1)$ \\
\hline $\mathrm{N} 2$ & Niijima & N1 & 14906 & $\begin{array}{l}34.21499^{\circ} \\
139.14331^{\circ}\end{array}$ & 0.6 & $0.4(0.8)$ & $\begin{array}{l}\text { Saccostrea } \\
\text { kegaki }\end{array}$ & $294 \pm 32$ & $\begin{array}{l}\text { Cal AD } \\
1847- \\
1950\end{array}$ & A & $0.2(-0.2)$ \\
\hline N3 & Niijima & N1 & 14907 & $\begin{array}{l}34.21499^{\circ} \\
139.14331^{\circ}\end{array}$ & 0.6 & $0.4(0.8)$ & $\begin{array}{l}\text { Saccostrea } \\
\text { kegaki }\end{array}$ & $-70 \pm 37$ & $\begin{array}{l}\text { Cal AD } \\
1885- \\
1950\end{array}$ & A & $0.2(-0.3)$ \\
\hline N4 & Niijima & N1 & 14908 & $\begin{array}{l}34.21499^{\circ} \\
139.14331^{\circ}\end{array}$ & 0.5 & $0.4(0.8)$ & $\begin{array}{l}\text { Saccostrea } \\
\text { kegaki }\end{array}$ & $-387 \pm 34$ & Modern & A & $0.1(-0.3)$ \\
\hline N5 & Niijima & N1 & 14909 & $\begin{array}{l}34.21499^{\circ} \\
139.14331^{\circ}\end{array}$ & 0.3 & $0.4(0.8)$ & $\begin{array}{l}\text { Saccostrea } \\
\text { kegaki }\end{array}$ & $-686 \pm 44$ & Modern & A & $-0.1(-0.5)$ \\
\hline N6 & Niijima & N1 & 14910 & $\begin{array}{l}34.21499^{\circ} \\
139.14331^{\circ}\end{array}$ & 0.3 & $0.4(0.8)$ & $\begin{array}{l}\text { Saccostrea } \\
\text { kegaki }\end{array}$ & $-687 \pm 46$ & Modern & A & $-0.1(-0.5)$ \\
\hline N7 & Niijima & N1 & 14911 & $\begin{array}{l}34.21499^{\circ} \\
139.14331^{\circ}\end{array}$ & 0.2 & $0.4(0.8)$ & $\begin{array}{l}\text { Saccostrea } \\
\text { kegaki }\end{array}$ & $-595 \pm 34$ & Modern & A & $-0.2(-0.6)$ \\
\hline N8 & Niijima & N1 & 14912 & $\begin{array}{l}34.21499^{\circ} \\
139.14331^{\circ}\end{array}$ & 0.1 & $0.4(0.8)$ & $\begin{array}{l}\text { Saccostrea } \\
\text { kegaki }\end{array}$ & $-720 \pm 48$ & Modern & A & $-0.3(-0.7)$ \\
\hline N9 & Niijima & N1 & 14913 & $\begin{array}{l}34.21499^{\circ} \\
139.14331^{\circ}\end{array}$ & 0.1 & $0.4(0.8)$ & $\begin{array}{l}\text { Saccostrea } \\
\text { kegaki }\end{array}$ & $-789 \pm 54$ & Modern & A & $-0.3(-0.7)$ \\
\hline N10 & Niijima & N1 & 14917 & $\begin{array}{l}34.21499^{\circ} \\
139.14331^{\circ}\end{array}$ & 0.1 & $0.4(0.8)$ & $\begin{array}{l}\text { Saccostrea } \\
\text { kegaki }\end{array}$ & $-815 \pm 56$ & Modern & A & $-0.3(-0.7)$ \\
\hline N11 & Niijima & N1 & 14918 & $\begin{array}{l}34.21499^{\circ} \\
139.14331^{\circ}\end{array}$ & 0.9 & $0.4(0.8)$ & $\begin{array}{l}\text { Crassostrea } \\
\text { gigas }\end{array}$ & $-1157 \pm 64$ & Modern & A & $0.5(0.1)$ \\
\hline $\mathrm{N} 12$ & Niijima & N1 & 14919 & $\begin{array}{l}34.21499^{\circ} \\
139.14331^{\circ}\end{array}$ & 0.9 & $0.4(0.8)$ & $\begin{array}{l}\text { Crassostrea } \\
\text { gigas }\end{array}$ & $-1088 \pm 42$ & Modern & A & $0.5(0.1)$ \\
\hline N13 & Niijima & N1 & 14920 & $\begin{array}{l}34.21499^{\circ} \\
139.14331^{\circ}\end{array}$ & 0.9 & $0.4(0.8)$ & $\begin{array}{l}\text { Crassostrea } \\
\text { gigas }\end{array}$ & $-1151 \pm 41$ & Modern & A & $0.5(0.1)$ \\
\hline $\mathrm{N} 14$ & Niijima & N1 & 14921 & $\begin{array}{l}34.21499^{\circ} \\
139.14331^{\circ}\end{array}$ & 0.9 & $0.4(0.8)$ & $\begin{array}{l}\text { Crassostrea } \\
\text { gigas }\end{array}$ & $-1171 \pm 46$ & Modern & A & $0.5(0.1)$ \\
\hline N15 & Niijima & N1 & 14922 & $\begin{array}{l}34.21499^{\circ} \\
139.14331^{\circ}\end{array}$ & 0.7 & $0.4(0.8)$ & $\begin{array}{l}\text { Crassostrea } \\
\text { gigas }\end{array}$ & $-1209 \pm 57$ & Modern & A & $0.3(-0.1)$ \\
\hline$J 11$ & Jinaijima & $\mathrm{J1}$ & 18537 & $\begin{array}{l}34.22027^{\circ} \\
139.13431^{\circ}\end{array}$ & 0.6 & 0.2 & $\begin{array}{l}\text { Pomatoleios } \\
\text { kraussii }\end{array}$ & $482 \pm 43$ & $\begin{array}{l}\text { Cal AD } \\
1724- \\
1950\end{array}$ & A & 0.4 \\
\hline $\mathrm{J} 12$ & Jinaijima & $\mathrm{J1}$ & 18538 & $\begin{array}{l}34.22027^{\circ} \\
139.13431^{\circ}\end{array}$ & 0.6 & 0.2 & $\begin{array}{l}\text { Pomatoleios } \\
\text { kraussii }\end{array}$ & $459 \pm 35$ & $\begin{array}{l}\text { Cal AD } \\
1726- \\
1950\end{array}$ & A & 0.4 \\
\hline TMR1 & Shikinejima & S1 & 9905 & $\begin{array}{l}34.20119^{\circ} \\
139.12376^{\circ}\end{array}$ & 4 & 0.8 & $\begin{array}{l}\text { Tetraclitella } \\
\text { chinensis }\end{array}$ & $1243 \pm 29$ & $\begin{array}{l}\text { Cal AD } \\
1139- \\
1391\end{array}$ & B & 3.2 \\
\hline TMR2 & Shikinejima & S1 & 14923 & $\begin{array}{l}34.20119^{\circ} \\
139.12376^{\circ}\end{array}$ & 4 & 0.8 & $\begin{array}{l}\text { Tetraclitella } \\
\text { chinensis }\end{array}$ & $955 \pm 64$ & $\begin{array}{l}\text { Cal AD } \\
1335- \\
1650\end{array}$ & B & 3.2 \\
\hline TMR3 & Shikinejima & S1 & 9906 & $\begin{array}{l}34.20119^{\circ} \\
139.12376^{\circ}\end{array}$ & 3.8 & 0.8 & $\begin{array}{l}\text { Tetraclitella } \\
\text { chinensis }\end{array}$ & $1270 \pm 35$ & $\begin{array}{l}\text { Cal AD } \\
1085- \\
1333\end{array}$ & B & 3 \\
\hline TMR4 & Shikinejima & S1 & 14924 & $\begin{array}{l}34.20119^{\circ} \\
139.12376^{\circ}\end{array}$ & 3.8 & 0.8 & $\begin{array}{l}\text { Tetraclitella } \\
\text { chinensis }\end{array}$ & $1006 \pm 60$ & $\begin{array}{l}\text { Cal AD } \\
1302- \\
1615\end{array}$ & B & 3 \\
\hline TMR5 & Shikinejima & S1 & 9907 & $\begin{array}{l}34.20119^{\circ} \\
139.12376^{\circ}\end{array}$ & 3.6 & 0.8 & $\begin{array}{l}\text { Tetraclitella } \\
\text { chinensis }\end{array}$ & $859 \pm 43$ & $\begin{array}{l}\text { Cal AD } \\
1460- \\
1670\end{array}$ & B & 2.8 \\
\hline
\end{tabular}


Table 2 Results of ${ }^{14} \mathrm{C}$ dating in the present study (Continued)

\begin{tabular}{|c|c|c|c|c|c|c|c|c|c|c|c|}
\hline $\begin{array}{l}\text { Sample } \\
\text { name }\end{array}$ & Locality & Site & $\begin{array}{l}\text { Laboratory } \\
\text { number } \\
\text { (YAUT-) }\end{array}$ & $\begin{array}{l}\text { Coordinates } \\
\text { (lat/long) }\end{array}$ & $\begin{array}{l}\text { Height } \\
\text { (m) }\end{array}$ & $\begin{array}{l}\text { Upper- } \\
\text { growth } \\
\text { limit (m) }\end{array}$ & Materials & $\begin{array}{l}\text { Conventional } \\
{ }^{14} \mathrm{C} \text { age (years } \\
\mathrm{BP} \text { ) }\end{array}$ & $\begin{array}{l}\text { Calibrated } \\
\text { age }(2 \sigma)\end{array}$ & Groups & $\begin{array}{l}\text { Total } \\
\text { minimum } \\
\text { uplift (m) }\end{array}$ \\
\hline TMR6 & Shikinejima & S1 & 14925 & $\begin{array}{l}34.20119^{\circ} \\
139.12376^{\circ}\end{array}$ & 3.6 & 0.8 & $\begin{array}{l}\text { Tetraclitella } \\
\text { chinensis }\end{array}$ & $1265 \pm 37$ & $\begin{array}{l}\text { Cal AD } \\
1069- \\
1380\end{array}$ & B & 2.8 \\
\hline TMR7 & Shikinejima & S1 & 9908 & $\begin{array}{l}34.20119^{\circ} \\
139.12376^{\circ}\end{array}$ & 3.4 & 0.8 & $\begin{array}{l}\text { Tetraclitella } \\
\text { chinensis }\end{array}$ & $1051 \pm 41$ & $\begin{array}{l}\text { Cal AD } \\
1308- \\
1487\end{array}$ & B & 2.6 \\
\hline TMR8 & Shikinejima & S1 & 14926 & $\begin{array}{l}34.20119^{\circ} \\
139.12376^{\circ}\end{array}$ & 3.4 & 0.8 & $\begin{array}{l}\text { Tetraclitella } \\
\text { chinensis }\end{array}$ & $837 \pm 70$ & $\begin{array}{l}\text { Cal AD } \\
1431- \\
1804\end{array}$ & B & 2.6 \\
\hline TMR9 & Shikinejima & S1 & 9909 & $\begin{array}{l}34.20119^{\circ} \\
139.12376^{\circ}\end{array}$ & 3.2 & 0.8 & $\begin{array}{l}\text { Tetraclitella } \\
\text { chinensis }\end{array}$ & $844 \pm 34$ & $\begin{array}{l}\text { Cal AD } \\
1468- \\
1678\end{array}$ & B & 2.4 \\
\hline TMR10 & Shikinejima & S1 & 14930 & $\begin{array}{l}34.20119^{\circ} \\
139.12376^{\circ}\end{array}$ & 3.2 & 0.8 & $\begin{array}{l}\text { Tetraclitella } \\
\text { chinensis }\end{array}$ & $1319 \pm 36$ & $\begin{array}{l}\text { Cal AD } \\
1048- \\
1305\end{array}$ & B & 2.4 \\
\hline TMR11 & Shikinejima & S1 & 9910 & $\begin{array}{l}34.20119^{\circ} \\
139.12376^{\circ}\end{array}$ & 3 & 0.8 & $\begin{array}{l}\text { Tetraclitella } \\
\text { chinensis }\end{array}$ & $904 \pm 40$ & $\begin{array}{l}\text { Cal AD } \\
1436- \\
1651\end{array}$ & B & 2.2 \\
\hline TMR12 & Shikinejima & S1 & 14931 & $\begin{array}{l}34.20119^{\circ} \\
139.12376^{\circ}\end{array}$ & 3 & 0.8 & $\begin{array}{l}\text { Tetraclitella } \\
\text { chinensis }\end{array}$ & $1117 \pm 47$ & $\begin{array}{l}\text { Cal AD } \\
1245- \\
1469\end{array}$ & B & 2.2 \\
\hline TMR13 & Shikinejima & S1 & 9911 & $\begin{array}{l}34.20119^{\circ} \\
139.12376^{\circ}\end{array}$ & 2.7 & 0.8 & $\begin{array}{l}\text { Tetraclitella } \\
\text { chinensis }\end{array}$ & $721 \pm 34$ & $\begin{array}{l}\text { Cal AD } \\
1539- \\
1872\end{array}$ & B & 1.9 \\
\hline TMR14 & Shikinejima & S1 & 14932 & $\begin{array}{l}34.20119^{\circ} \\
139.12376^{\circ}\end{array}$ & 2.7 & 0.8 & $\begin{array}{l}\text { Tetraclitella } \\
\text { chinensis }\end{array}$ & $774 \pm 43$ & $\begin{array}{l}\text { Cal AD } \\
1483- \\
1821\end{array}$ & B & 1.9 \\
\hline TMR15 & Shikinejima & S1 & 9912 & $\begin{array}{l}34.20119^{\circ} \\
139.12376^{\circ}\end{array}$ & 2.7 & 0.4 & Cardita leana & $1070 \pm 41$ & $\begin{array}{l}\text { Cal AD } \\
1300- \\
1473\end{array}$ & B & 2.3 \\
\hline TMR16 & Shikinejima & S1 & 14933 & $\begin{array}{l}34.20119^{\circ} \\
139.12376^{\circ}\end{array}$ & 2.7 & 0.4 & Cardita leana & $1378 \pm 38$ & $\begin{array}{l}\text { Cal AD } \\
1006- \\
1280\end{array}$ & B & 2.3 \\
\hline OUR22 & Shikinejima & S2 & 9917 & $\begin{array}{l}34.19534^{\circ} \\
139.12305^{\circ}\end{array}$ & 3 & 0.4 & Cardita leana & $1399 \pm 35$ & $\begin{array}{l}\text { Cal AD } \\
1002- \\
1252\end{array}$ & B & 2.6 \\
\hline OUR31 & Shikinejima & S3 & 9918 & $\begin{array}{l}34.19486^{\circ} \\
139.12267^{\circ}\end{array}$ & 2.3 & 0.8 & $\begin{array}{l}\text { Tetraclitella } \\
\text { chinensis }\end{array}$ & $1049 \pm 36$ & $\begin{array}{l}\text { Cal AD } \\
1309- \\
1489\end{array}$ & B & 1.5 \\
\hline OUR32 & Shikinejima & S3 & 9919 & $\begin{array}{l}34.19486^{\circ} \\
139.12267^{\circ}\end{array}$ & 2.3 & 0.8 & $\begin{array}{l}\text { Tetraclitella } \\
\text { chinensis }\end{array}$ & $1152 \pm 46$ & $\begin{array}{l}\text { Cal AD } \\
1216- \\
1452\end{array}$ & B & 1.5 \\
\hline NNU41 & Shikinejima & S4 & 9920 & $\begin{array}{l}34.19420^{\circ} \\
139.12238^{\circ}\end{array}$ & -0.1 & -0.5 & $\begin{array}{l}\text { Cyphastrea } \\
\text { serailia }\end{array}$ & $375 \pm 40$ & $\begin{array}{l}\text { Cal AD } \\
1842- \\
1950\end{array}$ & A & 0.4 \\
\hline NNU42 & Shikinejima & S4 & 9921 & $\begin{array}{l}34.19420^{\circ} \\
139.12238^{\circ}\end{array}$ & -0.1 & -0.5 & $\begin{array}{l}\text { Cyphastrea } \\
\text { serailia }\end{array}$ & $215 \pm 34$ & $\begin{array}{l}\text { Cal AD } \\
1875- \\
1950\end{array}$ & A & 0.4 \\
\hline NNU43 & Shikinejima & S4 & 9922 & $\begin{array}{l}34.19420^{\circ} \\
139.12238^{\circ}\end{array}$ & -0.1 & -0.5 & $\begin{array}{l}\text { Cyphastrea } \\
\text { serailia }\end{array}$ & $302 \pm 37$ & $\begin{array}{l}\text { Cal AD } \\
1857- \\
1950\end{array}$ & A & 0.4 \\
\hline NNU51 & Shikinejima & S5 & 9923 & $\begin{array}{l}34.19416^{\circ} \\
139.12198^{\circ}\end{array}$ & 1.4 & 0.8 & $\begin{array}{l}\text { Tetraclitella } \\
\text { chinensis }\end{array}$ & $275 \pm 29$ & $\begin{array}{l}\text { Cal AD } \\
1862- \\
1950\end{array}$ & A & 0.6 \\
\hline
\end{tabular}


Table 2 Results of ${ }^{14} \mathrm{C}$ dating in the present study (Continued)

\begin{tabular}{|c|c|c|c|c|c|c|c|c|c|c|c|}
\hline $\begin{array}{l}\text { Sample } \\
\text { name }\end{array}$ & Locality & Site & $\begin{array}{l}\text { Laboratory } \\
\text { number } \\
\text { (YAUT-) }\end{array}$ & $\begin{array}{l}\text { Coordinates } \\
\text { (lat/long) }\end{array}$ & $\begin{array}{l}\text { Height } \\
\text { (m) }\end{array}$ & $\begin{array}{l}\text { Upper- } \\
\text { growth } \\
\text { limit (m) }\end{array}$ & Materials & $\begin{array}{l}\text { Conventional } \\
{ }^{14} \mathrm{C} \text { age (years } \\
\mathrm{BP})\end{array}$ & $\begin{array}{l}\text { Calibrated } \\
\text { age }(2 \sigma)\end{array}$ & Groups & $\begin{array}{l}\text { Total } \\
\text { minimum } \\
\text { uplift (m) }\end{array}$ \\
\hline$\overline{N N U 51}$ & Shikinejima & S5 & 14936 & $\begin{array}{l}34.19416^{\circ} \\
139.12198^{\circ}\end{array}$ & 1.4 & 0.8 & $\begin{array}{l}\text { Tetraclitella } \\
\text { chinensis }\end{array}$ & $539 \pm 41$ & $\begin{array}{l}\text { Cal AD } \\
1720- \\
1950\end{array}$ & $A$ & 0.6 \\
\hline ISZ61 & Shikinejima & S6 & 9924 & $\begin{array}{l}34.19196^{\circ} \\
139.13216^{\circ}\end{array}$ & 3.7 & 0.4 & Cardita leana & $1260 \pm 38$ & $\begin{array}{l}\text { Cal AD } \\
1090- \\
1346\end{array}$ & B & 3.3 \\
\hline K111 & Kozujima & K1 & 18504 & $\begin{array}{l}34.14110^{\circ} \\
139.08083^{\circ}\end{array}$ & 0.54 & 0.4 & Tetraclita sp. & $-796 \pm 41$ & modern & A & 0.14 \\
\hline K112 & Kozujima & K1 & 18505 & $\begin{array}{l}34.14110^{\circ} \\
139.08083^{\circ}\end{array}$ & 0.64 & 0.8 & $\begin{array}{l}\text { Serpulidae } \\
\text { sp. }\end{array}$ & $439 \pm 29$ & $\begin{array}{l}\text { Cal AD } \\
1766- \\
1950\end{array}$ & A & -0.16 \\
\hline K113 & Kozujima & K1 & 18506 & $\begin{array}{l}34.14110^{\circ} \\
139.08083^{\circ}\end{array}$ & 0.99 & 0.4 & Tetraclita sp. & $472 \pm 27$ & $\begin{array}{l}\text { Cal AD } \\
1727- \\
1950\end{array}$ & A & 0.59 \\
\hline K114 & Kozujima & K1 & 18507 & $\begin{array}{l}34.14110^{\circ} \\
139.08083^{\circ}\end{array}$ & 1.36 & 0.4 & Tetraclita sp. & $486 \pm 39$ & $\begin{array}{l}\text { Cal AD } \\
1724- \\
1950\end{array}$ & A & 0.96 \\
\hline K121 & Kozujima & K1 & 18509 & $\begin{array}{l}34.14110^{\circ} \\
139.08083^{\circ}\end{array}$ & 2.42 & 0.8 & $\begin{array}{l}\text { Tetraclitella } \\
\text { chinensis }\end{array}$ & $1227 \pm 34$ & $\begin{array}{l}\text { Cal AD } \\
1140- \\
1410\end{array}$ & B & 1.62 \\
\hline K122 & Kozujima & K1 & 18511 & $\begin{array}{l}34.14110^{\circ} \\
139.08083^{\circ}\end{array}$ & 2.98 & 0.8 & $\begin{array}{l}\text { Tetraclitella } \\
\text { chinensis }\end{array}$ & $1280 \pm 30$ & $\begin{array}{l}\text { Cal AD } \\
1066- \\
1331\end{array}$ & B & 2.18 \\
\hline K123 & Kozujima & K1 & 18512 & $\begin{array}{l}34.14110^{\circ} \\
139.08083^{\circ}\end{array}$ & 3.28 & 0 & $\begin{array}{l}\text { Tubastraea } \\
\text { coccinea }\end{array}$ & $1414 \pm 28$ & $\begin{array}{l}\text { Cal AD } \\
975-1255\end{array}$ & B & 3.28 \\
\hline K131 & Kozujima & K1 & 18513 & $\begin{array}{l}34.14110^{\circ} \\
139.08083^{\circ}\end{array}$ & 4.36 & 0.8 & $\begin{array}{l}\text { Rhizotrochus } \\
\text { typus }\end{array}$ & $1626 \pm 45$ & $\begin{array}{l}\text { Cal AD } \\
716-1031\end{array}$ & C & 3.56 \\
\hline K132 & Kozujima & K1 & 18514 & $\begin{array}{l}34.14110^{\circ} \\
139.08083^{\circ}\end{array}$ & 4.91 & 0.8 & $\begin{array}{l}\text { Rhizotrochus } \\
\text { typus }\end{array}$ & $1663 \pm 57$ & $\begin{array}{l}\text { Cal AD } \\
682-1015\end{array}$ & C & 4.11 \\
\hline K133 & Kozujima & K1 & 18517 & $\begin{array}{l}34.14110^{\circ} \\
139.08083^{\circ}\end{array}$ & 5.58 & 0.8 & $\begin{array}{l}\text { Rhizotrochus } \\
\text { typus }\end{array}$ & $1594 \pm 42$ & $\begin{array}{l}\text { Cal AD } \\
735-1053\end{array}$ & C & 4.78 \\
\hline K141 & Kozujima & K1 & 18518 & $\begin{array}{l}34.14110^{\circ} \\
139.08083^{\circ}\end{array}$ & 6.2 & 0.8 & $\begin{array}{l}\text { Rhizotrochus } \\
\text { sp. }\end{array}$ & $1725 \pm 42$ & $\begin{array}{l}\text { Cal AD } \\
647-956\end{array}$ & C & 5.4 \\
\hline K142 & Kozujima & K1 & 18519 & $\begin{array}{l}34.14110^{\circ} \\
139.08083^{\circ}\end{array}$ & 6.91 & 0.8 & $\begin{array}{l}\text { Rhizotrochus } \\
\text { typus }\end{array}$ & $1663 \pm 33$ & $\begin{array}{l}\text { Cal AD } \\
696-993\end{array}$ & $C$ & 6.11 \\
\hline K143 & Kozujima & K1 & 18521 & $\begin{array}{l}34.14110^{\circ} \\
139.08083^{\circ}\end{array}$ & 6.91 & 0 & $\begin{array}{l}\text { Tubastraea } \\
\text { coccinea }\end{array}$ & $1568 \pm 29$ & $\begin{array}{l}\text { Cal AD } \\
775-1073\end{array}$ & C & 6.91 \\
\hline K1441 & Kozujima & K1 & 18523 & $\begin{array}{l}34.14110^{\circ} \\
139.08083^{\circ}\end{array}$ & 6.61 & 0.8 & $\begin{array}{l}\text { Rhizotrochus } \\
\text { typus }\end{array}$ & $1503 \pm 32$ & $\begin{array}{l}\text { Cal AD } \\
843-1173\end{array}$ & C & 5.81 \\
\hline K1442 & Kozujima & K1 & 22004 & $\begin{array}{l}34.14110^{\circ} \\
139.08083^{\circ}\end{array}$ & 6.61 & 0 & $\begin{array}{l}\text { Chama } \\
\text { iostoma }\end{array}$ & $1639 \pm 33$ & $\begin{array}{l}\text { Cal AD } \\
715-1015\end{array}$ & C & 6.61 \\
\hline K145 & Kozujima & K1 & 18524 & $\begin{array}{l}34.14110^{\circ} \\
139.08083^{\circ}\end{array}$ & 6.51 & 0.8 & $\begin{array}{l}\text { Rhizotrochus } \\
\text { typus }\end{array}$ & $1537 \pm 51$ & $\begin{array}{l}\text { Cal AD } \\
786-1154\end{array}$ & C & 5.71 \\
\hline K146 & Kozujima & K1 & 18525 & $\begin{array}{l}34.14110^{\circ} \\
139.08083^{\circ}\end{array}$ & 6.51 & 0 & $\begin{array}{l}\text { Tubastraea } \\
\text { coccinea }\end{array}$ & $1779 \pm 67$ & $\begin{array}{l}\text { Cal AD } \\
550-940\end{array}$ & C & 6.51 \\
\hline K211 & Kozujima & K2 & 18526 & $\begin{array}{l}34.13131^{\circ} \\
139.07457^{\circ}\end{array}$ & 9.43 & 0 & Tetraclita sp. & $1931 \pm 33$ & $\begin{array}{l}\text { Cal AD } \\
426-700\end{array}$ & $\mathrm{D}$ & 9.43 \\
\hline K212 & Kozujima & K2 & 22005 & $\begin{array}{l}34.13131^{\circ} \\
139.07457^{\circ}\end{array}$ & 9.43 & 0.4 & Cardita leana & $2085 \pm 29$ & $\begin{array}{l}\text { Cal AD } \\
\text { 250-585 }\end{array}$ & $\mathrm{D}$ & 9.03 \\
\hline K213 & Kozujima & K2 & 22006 & $\begin{array}{l}34.13131^{\circ} \\
139.07457^{\circ}\end{array}$ & 9.43 & 0.8 & $\begin{array}{l}\text { Lithophaga } \\
\text { curta }\end{array}$ & $2035 \pm 36$ & $\begin{array}{l}\text { Cal AD } \\
\text { 305-641 }\end{array}$ & $\mathrm{D}$ & 8.63 \\
\hline K214 & Kozujima & K2 & 22007 & $\begin{array}{l}34.13131^{\circ} \\
139.07457^{\circ}\end{array}$ & 9.43 & 0 & Tetraclita sp. & $1982 \pm 25$ & $\begin{array}{l}\text { Cal AD } \\
395-665\end{array}$ & $\mathrm{D}$ & 9.43 \\
\hline
\end{tabular}


Table 2 Results of ${ }^{14} \mathrm{C}$ dating in the present study (Continued)

\begin{tabular}{|c|c|c|c|c|c|c|c|c|c|c|c|}
\hline $\begin{array}{l}\text { Sample } \\
\text { name }\end{array}$ & Locality & Site & $\begin{array}{l}\text { Laboratory } \\
\text { number } \\
\text { (YAUT-) }\end{array}$ & $\begin{array}{l}\text { Coordinates } \\
\text { (lat/long) }\end{array}$ & $\begin{array}{l}\text { Height } \\
(\mathrm{m})\end{array}$ & $\begin{array}{l}\text { Upper- } \\
\text { growth } \\
\text { limit (m) }\end{array}$ & Materials & $\begin{array}{l}\text { Conventional } \\
{ }^{14} \mathrm{C} \text { age (years } \\
\mathrm{BP} \text { ) }\end{array}$ & $\begin{array}{l}\text { Calibrated } \\
\text { age }(2 \sigma)\end{array}$ & Groups & $\begin{array}{l}\text { Total } \\
\text { minimum } \\
\text { uplift }(\mathrm{m})\end{array}$ \\
\hline K215 & Kozujima & K2 & 22009 & $\begin{array}{l}34.13131^{\circ} \\
139.07457^{\circ}\end{array}$ & 9.43 & -0.4 & $\begin{array}{l}\text { Vermetus } \\
\text { tokyoensis }\end{array}$ & $2107 \pm 35$ & $\begin{array}{l}\text { Cal AD } \\
218-572\end{array}$ & D & 9.83 \\
\hline K22 & Kozujima & $\mathrm{K} 2$ & 18527 & $\begin{array}{l}34.13131^{\circ} \\
139.07457^{\circ}\end{array}$ & 11.43 & 0.4 & Cardita leana & $2156 \pm 47$ & $\begin{array}{l}\text { Cal AD } \\
142-528\end{array}$ & D & 11.03 \\
\hline K31 & Kozujima & K3 & 18531 & $\begin{array}{l}34.12475^{\circ} \\
139.07517^{\circ}\end{array}$ & 2.09 & 0.8 & $\begin{array}{l}\text { Serpulidae } \\
\text { sp. }\end{array}$ & $1301 \pm 35$ & $\begin{array}{l}\text { Cal AD } \\
1056- \\
1316\end{array}$ & B & 1.29 \\
\hline K321 & Kozujima & K3 & 18532 & $\begin{array}{l}34.12475^{\circ} \\
139.07517^{\circ}\end{array}$ & 2.45 & 0.4 & Cardita leana & $1789 \pm 40$ & $\begin{array}{l}\text { Cal AD } \\
580-885\end{array}$ & C & 2.05 \\
\hline K322 & Kozujima & K3 & 22011 & $\begin{array}{l}34.12475^{\circ} \\
139.07517^{\circ}\end{array}$ & 2.45 & -0.4 & $\begin{array}{l}\text { Vermetus } \\
\text { tokyoensis }\end{array}$ & $1617 \pm 39$ & $\begin{array}{l}\text { Cal AD } \\
726-1034\end{array}$ & C & 2.85 \\
\hline K323 & Kozujima & K3 & 22012 & $\begin{array}{l}34.12475^{\circ} \\
139.07517^{\circ}\end{array}$ & 2.45 & 0.8 & $\begin{array}{l}\text { Lithophaga } \\
\text { curta }\end{array}$ & $1553 \pm 31$ & $\begin{array}{l}\text { Cal AD } \\
783-1100\end{array}$ & C & 1.65 \\
\hline K33 & Kozujima & K3 & 22013 & $\begin{array}{l}34.12475^{\circ} \\
139.07517^{\circ}\end{array}$ & 1.22 & 0.4 & Tetraclita sp. & $480 \pm 32$ & $\begin{array}{l}\text { Cal AD } \\
1725- \\
1950\end{array}$ & A & 0.82 \\
\hline K411 & Kozujima & K4 & 18534 & $\begin{array}{l}34.12462^{\circ} \\
139.07516^{\circ}\end{array}$ & 0.02 & 0.8 & $\begin{array}{l}\text { Serpulidae } \\
\text { sp. }\end{array}$ & $-816 \pm 34$ & Modern & A & -0.78 \\
\hline K4121 & Kozujima & K4 & 18536 & $\begin{array}{l}34.12462^{\circ} \\
139.07516^{\circ}\end{array}$ & 0.32 & 0.8 & $\begin{array}{l}\text { Serpulidae } \\
\text { sp. }\end{array}$ & $-923 \pm 46$ & Modern & A & -0.48 \\
\hline K4122 & Kozujima & K4 & 22014 & $\begin{array}{l}34.12462^{\circ} \\
139.07516^{\circ}\end{array}$ & 0.32 & 0.4 & Tetraclita sp. & $-1121 \pm 23$ & Modern & A & 0.32 \\
\hline K413 & Kozujima & K4 & 18636 & $\begin{array}{l}34.12462^{\circ} \\
139.07516^{\circ}\end{array}$ & 0.62 & 0.8 & $\begin{array}{l}\text { Dendropoma } \\
\text { planorbis }\end{array}$ & $530 \pm 31$ & $\begin{array}{l}\text { Cal AD } \\
1723- \\
1950\end{array}$ & A & -0.18 \\
\hline K421 & Kozujima & K4 & 18637 & $\begin{array}{l}34.12462^{\circ} \\
139.07516^{\circ}\end{array}$ & 2.02 & 0.8 & $\begin{array}{l}\text { Tetraclitella } \\
\text { chinensis }\end{array}$ & $1037 \pm 28$ & $\begin{array}{l}\text { Cal AD } \\
1306-1511\end{array}$ & B & 1.22 \\
\hline K422 & Kozujima & K4 & 18638 & $\begin{array}{l}34.12462^{\circ} \\
139.07516^{\circ}\end{array}$ & 3.92 & 0.8 & $\begin{array}{l}\text { Serpulidae } \\
\text { sp. }\end{array}$ & $1755 \pm 28$ & $\begin{array}{l}\text { Cal AD } \\
626-901\end{array}$ & $C$ & 3.12 \\
\hline $\begin{array}{l}\text { GaK- } \\
9718^{*}\end{array}$ & Shikinejima & $\begin{array}{l}1 \\
(\mathrm{~S} 1)\end{array}$ & GaK-9718 & $\begin{array}{l}34.20119^{\circ} \\
139.12376^{\circ}\end{array}$ & $3.2-3.6$ & 0.8 & $\begin{array}{l}\text { Tetraclitella } \\
\text { chinensi }\end{array}$ & $1980 \pm 130$ & & & $2.4-2.8$ \\
\hline $\begin{array}{l}\text { GaK- } \\
9717^{*}\end{array}$ & Shikinejima & $\begin{array}{l}1 \\
(\mathrm{~S} 1)\end{array}$ & GaK-9717 & $\begin{array}{l}34.20119^{\circ} \\
139.12376^{\circ}\end{array}$ & $3.0-3.1$ & 0.8 & $\begin{array}{l}\text { Megabalanus } \\
\text { rosa }\end{array}$ & $1830 \pm 130$ & & & $2.2-2.3$ \\
\hline $\begin{array}{l}\text { GaK- } \\
9720^{*}\end{array}$ & Shikinejima & $\begin{array}{l}1 \\
(\mathrm{~S} 1)\end{array}$ & GaK-9720 & $\begin{array}{l}34.20119^{\circ} \\
139.12376^{\circ}\end{array}$ & 2.6 & 0.8 & $\begin{array}{l}\text { Tetraclitella } \\
\text { chinensi }\end{array}$ & $1350 \pm 100$ & & & 1.8 \\
\hline $\begin{array}{l}\text { GaK- } \\
9721^{*}\end{array}$ & Shikinejima & $\begin{array}{l}1 \\
(\mathrm{~S} 1)\end{array}$ & GaK-9721 & $\begin{array}{l}34.20119^{\circ} \\
139.12376^{\circ}\end{array}$ & 2.6 & 0.8 & $\begin{array}{l}\text { Saccostrea } \\
\text { kegaki }\end{array}$ & $2540 \pm 290$ & & & 1.8 \\
\hline $\begin{array}{l}\text { GaK- } \\
9719^{*}\end{array}$ & Shikinejima & 2 & GaK-9719 & $\begin{array}{l}34.19165^{\circ} \\
139.13214^{\circ}\end{array}$ & 4.2 & 0.8 & $\begin{array}{l}\text { Tetraclita } \\
\text { japonica }\end{array}$ & $1010 \pm 300$ & & & 3.4 \\
\hline $\begin{array}{l}\text { GaK- } \\
9722^{*}\end{array}$ & Shikinejima & 3 & GaK-9722 & $\begin{array}{l}34.19184^{\circ} \\
139.13223^{\circ}\end{array}$ & $3.9-5.1$ & 0.8 & $\begin{array}{l}\text { Tetraclitella } \\
\text { chinensi }\end{array}$ & $1530 \pm 100$ & & & $3.1-4.3$ \\
\hline
\end{tabular}

*Ota et al. (1983)

Their ${ }^{14} \mathrm{C}$ ages exactly matched each other and ranged from AD 1820 to present (Table 2). Observations made while scuba diving indicate that living hermatypic corals at this site occupy water depths greater than $0.5 \mathrm{~m}$.

Site S5 is a marine cave located in a cliff on the east side of Nakanoura Bay. The cave is $1.8 \mathrm{~m}$ wide, $3.0 \mathrm{~m}$ high, and $6.0 \mathrm{~m}$ long. We collected two well-preserved individual specimens of $T$. chinensis from inside the cave at an elevation of $1.8 \mathrm{~m}$ amsl. Their ${ }^{14} \mathrm{C}$ ages fall within $\mathrm{AD}$ 1720 to present (Table 2).
Site S6 is located on bedrock on the Ishijirogawa coast, at about $+3.7 \mathrm{~m}$ amsl. One specimen of $C$. leana was collected and yielded a ${ }^{14} \mathrm{C}$ age of AD 1077-1385 (Table 2).

\section{Kouzushima}

Site K1 is located on the southern side of Nagumi Bay and is divided into four subsites (K11, K12, K13, and K14) (Figs. 2 and 9). Well-preserved individuals of Tetraclita sp., Serpulidae sp., and T. chinensis were observed at $0.5-3.0 \mathrm{~m}$ amsl on coastal bedrock at sites K11 and K12 (Table 2). We 

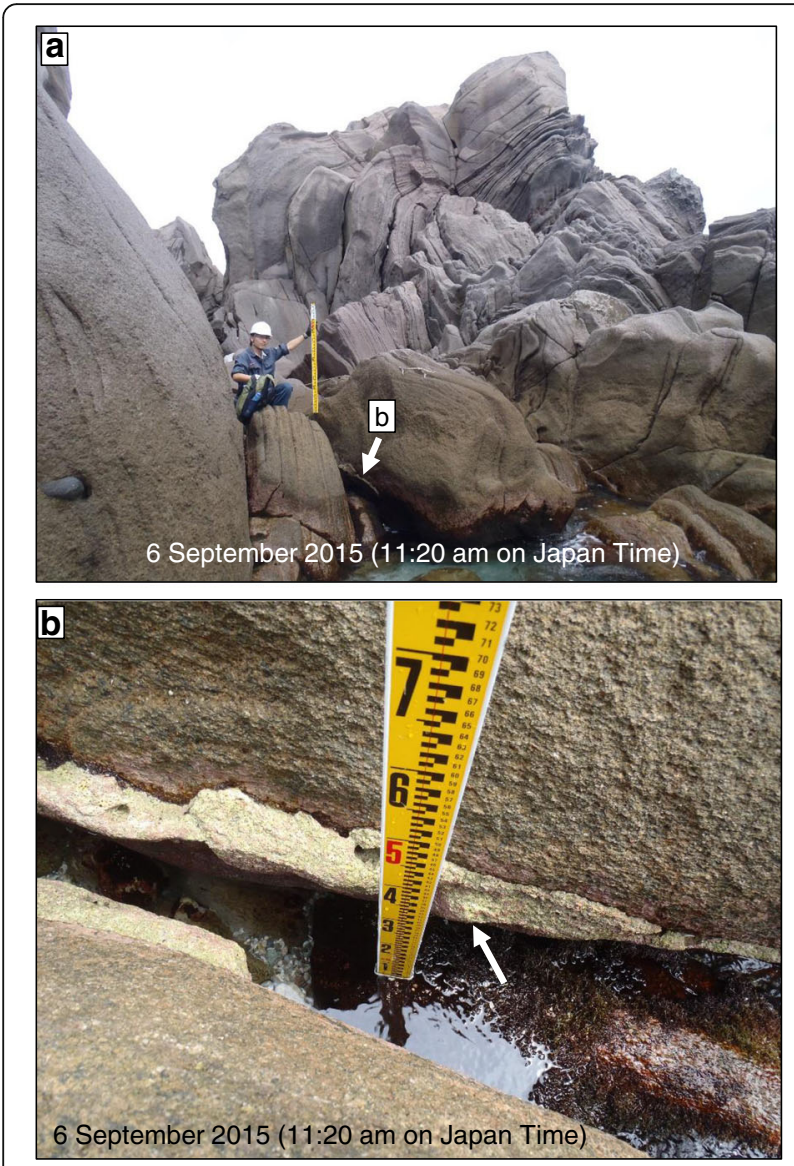

Fig. 6 Photographs of $\mathbf{a}$ site J1 on Jinaijima and $\mathbf{b}$ encrusting masses of $P$. kraussii collected many well-preserved in situ azooxanthellate corals of Tubastraea coccinea and Rhizotrochus typus at 3.3$6.9 \mathrm{~m}$ amsl from sites K13 and K14 (Fig. 9; Table 2) but did not find intertidal organisms such as $T$. chinensis and $S$. kegaki. Although many studies of emerged Holocene marine fossils have been performed in Japan (e.g., Ishibashi et al. 1979; Ota et al. 1983; Kayanne et al. 1987; Maemoku 1988; Miyauchi 1990; Iryu et al. 2009; Kitamura et al. 2014, 2015, 2016, in press), no previous reports of the occurrence of $R$. typus are known to the authors. All of the coral specimens collected during the present study are $100 \%$ aragonite and therefore are not susceptible to subaerial diagenesis, because recrystallization of coral aragonite to calcite occurs in a subaerial setting (Sayani et al. 2011). The ${ }^{14} \mathrm{C}$ ages of specimens at this site range from $\mathrm{AD} 613$ to present and become older with increasing elevation (Table 2).

Site K2 is located at 9.4-11.4 $\mathrm{m}$ amsl on bedrock on the northwest side of Sawajiri Bay (Figs. 2 and 10). At this location, samples were taken at the highest elevation of any site. We collected well-preserved Tetraclita sp., C. leana, Lithophaga curta, and Vermetus tokyoensis. Their ${ }^{14} \mathrm{C}$ ages fall within the range $\mathrm{AD} 161-686$ (Table 2).

Site K3 is located on bedrock north of Kouzushima Harbor, at 1.2-2.5 m amsl (Fig. 2). Well-preserved Serpulidae sp., C. leana, $V$. tokyoensis, and $L$. curta were collected with ${ }^{14} \mathrm{C}$ ages ranging from $\mathrm{AD} 600$ to the present (Table 2).

Site K4 is located on bedrock south of Maehama (Fig. 2). This site yielded individuals of Serpulidae sp., Tetraclita sp., Dendropoma planorbis, and T. chinensis at $0-3.9 \mathrm{~m}$ amsl. The ${ }^{14} \mathrm{C}$ ages of the specimens range between $\mathrm{AD}$ 626 and the present day and become older with increasing elevation (Table 2).
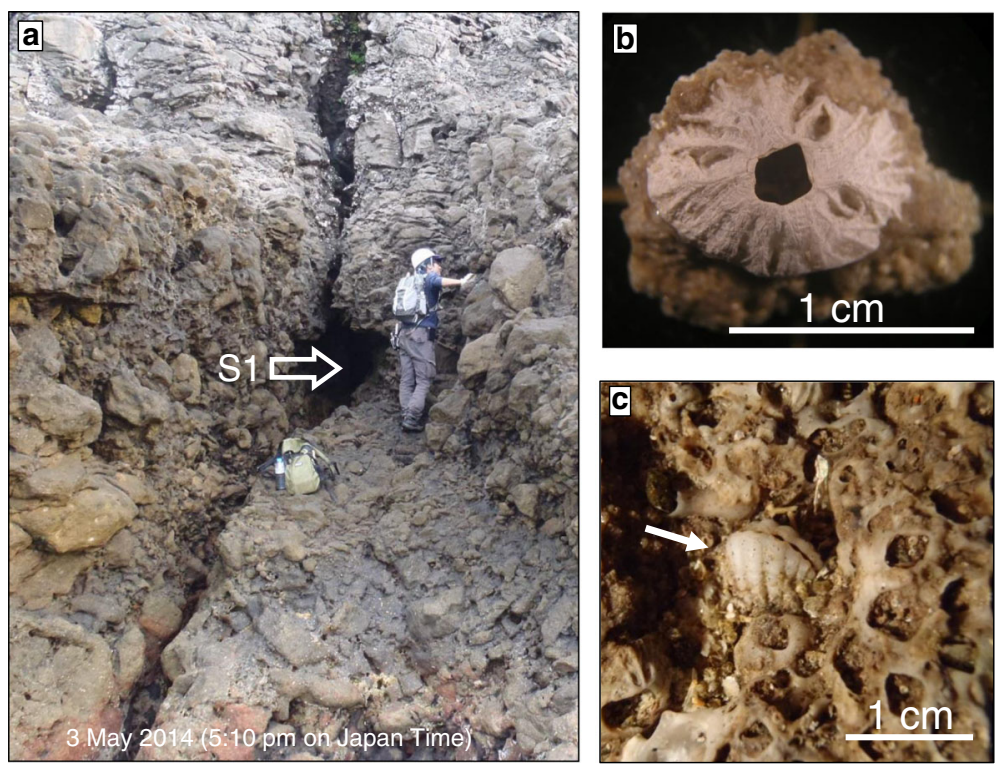

Fig. 7 Photographs of a site S1 on Shikinejima, $\mathbf{b}$ close-up view of a specimen of T. chinensis (sample TMR1), and $\mathbf{c}$ the in situ bivalve C. leana (sample TMR18) 

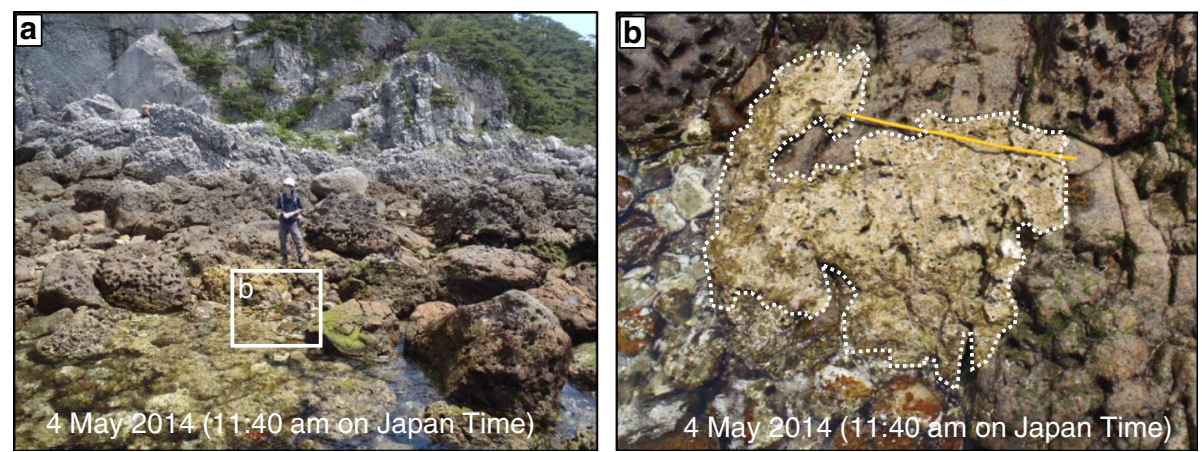

Fig. 8 Photographs of a site S4 on Shikinejima and $\mathbf{b}$ in situ colonies of hermatypic C. serailia (sample NNU41)

\section{Emerged sessile assemblages}

Figure 11 shows the elevations and ages $(2 \sigma)$ of emerged sessile assemblages from this study and from Ota et al. (1983). Rather than a simple linear trend, the data show stepwise changes in elevations at particular times, meaning that uplift occurred in a series of discrete events rather than as a gradual and continuous process. The mechanism of such uplift is a seismic event or relatively short-lived uplift associated with volcanic activity, such as the AD 2000 eruption of Miyakejima volcano (Fig. 4).

Based on these elevations and ages, the Shikinejima specimens cluster into two groups (A and B; Fig. 11 and Table 2). Group A occurs between -0.1 and $1.4 \mathrm{~m}$ amsl at Shikinejima (sites S4 and S5), Niijima (site N1), Jinaijima (site J1), and Kouzushima (sites K1, K3, and K4). The ages of the Group A specimens range from $\mathrm{AD} 1723$ to present. Group B samples are defined as those taken at elevations above Group A and are located between 2.0 and $4.0 \mathrm{~m}$ amsl at Shikinejima (sites S1, S2, S3, and S6) and Kouzushima (sites K1, K3, and K4); they yield ages of AD 975-1872. Groups C and D are only found on Kouzushima (sites K14). Samples in Group $C$ have elevations between those of Groups B and D. Although the elevations of four specimens (K321, K322, K323, and K422) fall within the range defined for Group B, their ages are consistent with other Group C samples, and thus, they are considered to belong to Group C. Groups C and D occur at elevations of 2.45-6.91 m amsl and 9.43-11.43 m amsl, respectively (Fig. 11), with no overlap in elevation. The corresponding age ranges for Groups $\mathrm{C}$ and $\mathrm{D}$ are $\mathrm{AD}$ 600-1165 and AD 161-686, respectively.

\section{Discussion}

All of the collected specimens are more recent than $\mathrm{AD}$ 160 (Fig. 11). Since relative sea-level reconstructions show that sea level was likely stable at the study site during the past 2000 years (Sato 2008; Woodroffe et al. 2012; Yokoyama et al. 2012; Tanigawa et al. 2013; Tanabe et al. 2016), the occurrence of the emerged sessile assemblages cannot be explained by eustatic sea level changes; instead, it requires local crustal uplift.
The magnitude of the uplift is best estimated by direct measurement of the elevational difference between the upper limit of the uplifted remains and the corresponding upper limit of its present homolog (Laborel and LaborelDeguen 1994; Pirazzoli et al. 1999). Although the estimated values represent the minimum amount of uplift, this procedure eliminates uncertainties derived from estimates of the bathymetric ranges of each species. If this procedure was not applied, we would have to make a subjective decision about whether any individuals of a species lived higher than the collected specimens but were not preserved. We therefore make this assumption since there is no reliable way of resolving this ambiguity. This estimation method was also used by Pirazzoli et al. (1999), Kitamura et al. (2014, 2015, 2016), and Hamada et al. (2016).

Using the above procedure, we calculated the uplift amount of each specimen. As noted below, the maximum value of each group was derived for each island from specimens found at the highest position in the group. The estimated maximum uplift was regarded as rapid uplift, based on the following conceptual assumptions: (1) each group of emerged assemblages was created by a single uplift event or by gradual uplift over a finite period of time and (2) no vertical crustal motion occurred in the study area during the intervals between discrete uplift events. These assumptions mean that our definition of an uplift event includes both coseismic uplift and relatively short-lived uplift due to volcanic activity. The estimated minimum total amount of uplift for each specimen is shown in Table 2. The defining events of each of Groups A-D are referred to (in reverse chronological order) as uplift events $1,2,3$, and 4 , respectively.

\section{Uplift event 1}

Group A consists of C. gigas and S. kegaki at Niijima Island, $P$. kraussii at Jinaijima, $T$. chinensis and C. serailia at Shikinejima, and D. planorbis, Serpulidae sp., and Tetraclita sp. at Kouzushima (Table 2).

C. gigas lives in intertidal and subtidal zones, while S. kegaki inhabits the intertidal zone at elevations 


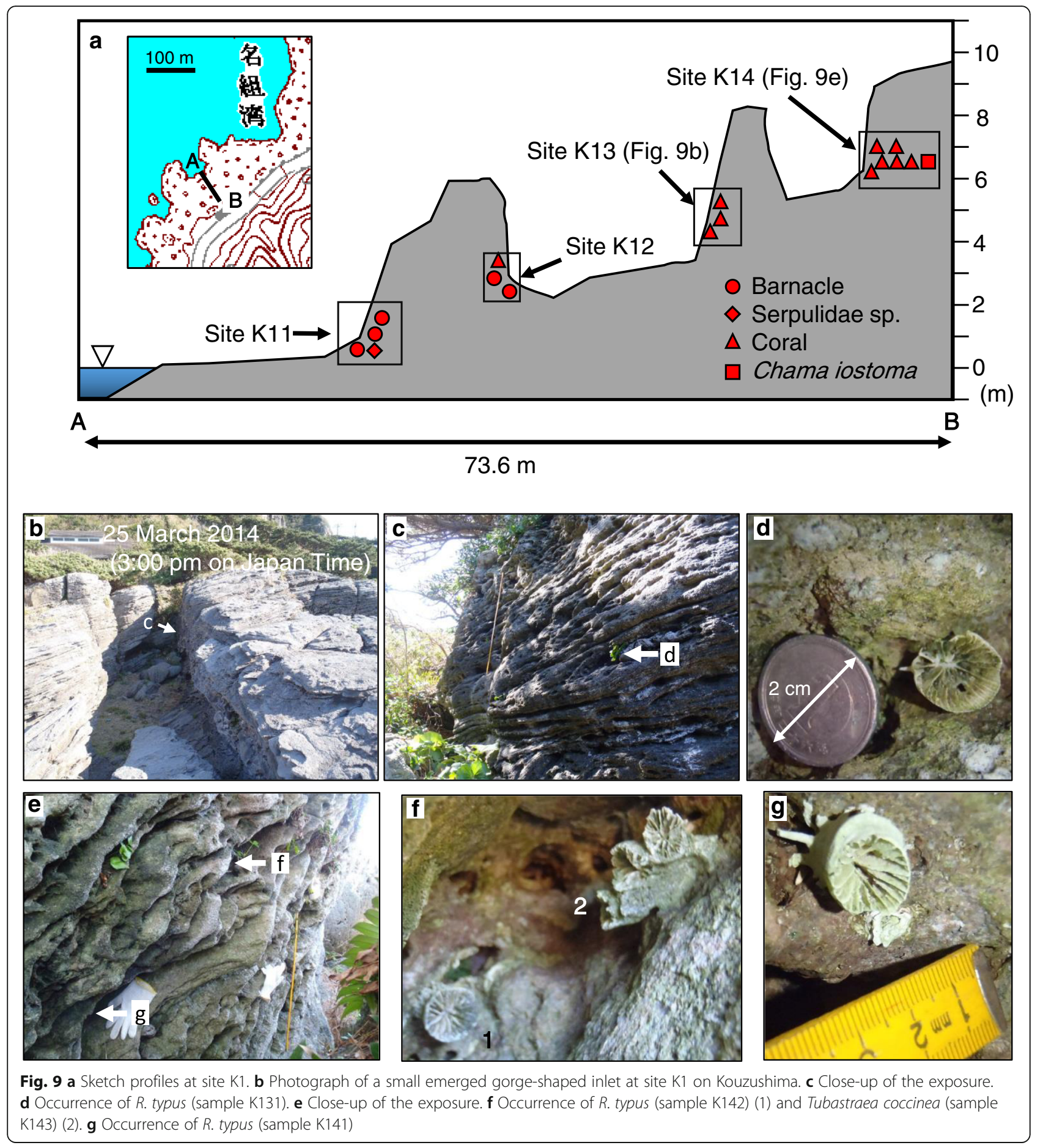

from -0.8 to $+0.8 \mathrm{~m}$ amsl (Okutani 2000). As noted above, since the upper limit of living $S$. kegaki is located at $+0.4 \mathrm{~m}$ amsl, we used both $+0.8 \mathrm{~m}$ (Okutani 2000) and $+0.4 \mathrm{~m}$ (this study) amsl as the upper limit (Table 2). Because the upper limit of $C$. gigas, which is absent in the study site, is the same as that of $S$. kegaki, the values +0.8 and $+0.4 \mathrm{~m}$ amsl were used for C. gigas.
The upper limit of encrustation by abundant calcareous tubes of living $P$. kraussii is located at an elevation of $0.1 \pm 0.1 \mathrm{~m}$ amsl on Boso Peninsula in Central Japan (Kayanne et al. 1987) and at 0.1-0.2 m amsl on Izu Peninsula in the same region (Kitamura et al. 2014). The value of $0.2 \mathrm{~m}$ amsl is used as the upper limit of encrustation of $P$. kraussii because we did not find living individuals in the study area. Local disappearances of $C$. 

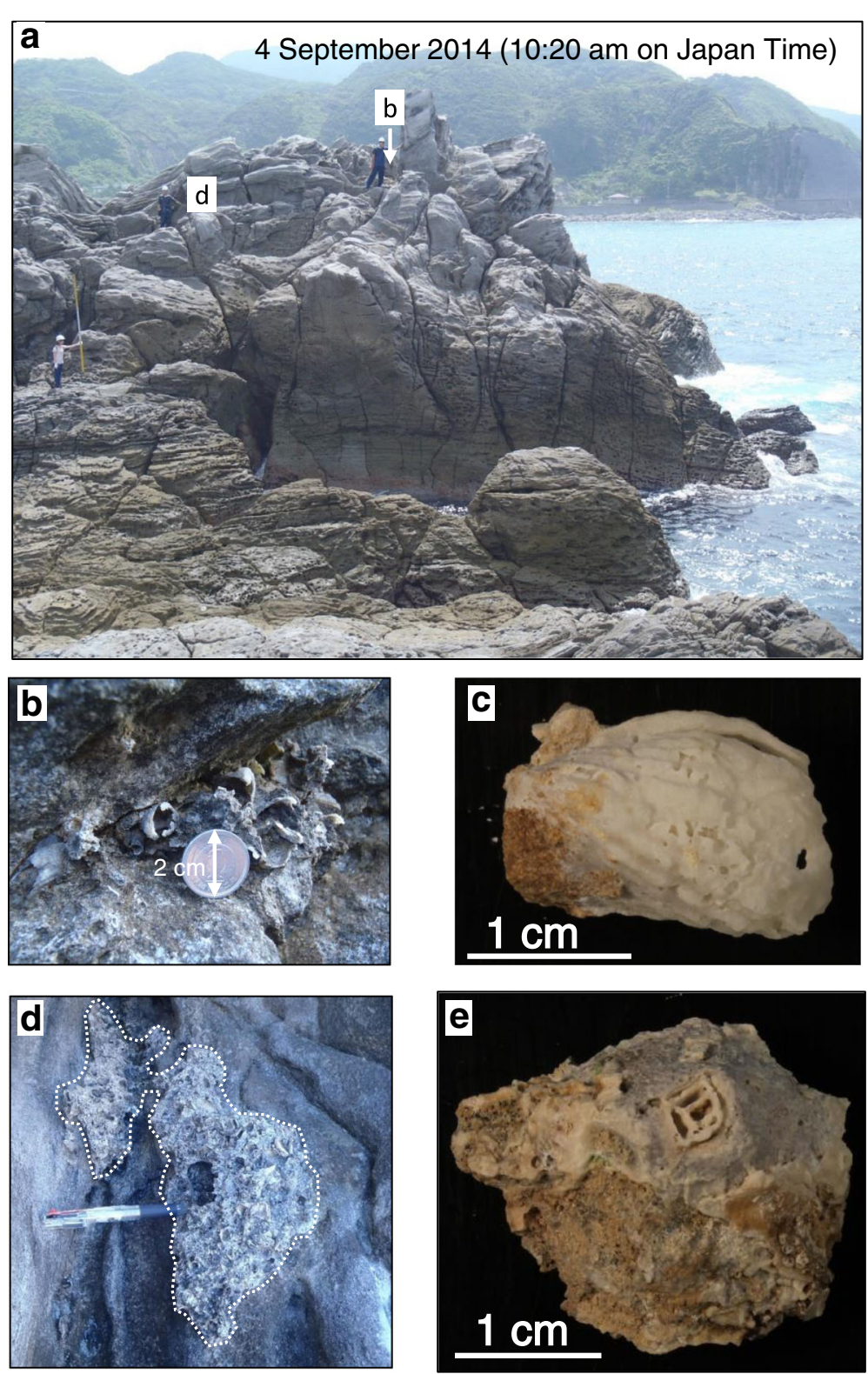

Fig. 10 Photographs of a site K2 on Kouzushima, $\mathbf{b}$ the emerged sessile assemblages, c specimen of C. leana (sample K22), $\mathbf{d}$ the emerged sessile assemblages, and e specimen of Tetraclita sp. (sample K211)

gigas and P. kraussii indicate that uplift of the area has made it an unsuitable environment for these species. Identical phenomena were documented in the southern Izu Peninsula by Kitamura et al. (2014).

T. chinensis and D. planorbis inhabit the intertidal zone from -0.8 to $+0.8 \mathrm{~m}$ amsl (Okutani 2000; Yamaguchi and Hisatsune 2006). This is consistent with our observation that the upper limit of $T$. chinensis is located at about $+0.8 \mathrm{~m}$ amsl. Tetraclita sp. lives around the middle tidal zone (Yamaguchi and Hisatsune 2006).

At site S4, where the specimens of $C$. serailia were found, our observations while scuba diving indicate that living colonies of $C$. serailia only inhabit water depths greater than $0.5 \mathrm{~m}$ below mean sea level.

Based on the vertical distribution of these living species, uplift event 1 is estimated to have a minimum uplift of $0.5 \mathrm{~m}$ at Niijima (samples N11-N14), $0.4 \mathrm{~m}$ at Jinaijima (J1), $0.6 \mathrm{~m}$ at Shikinejima (sample NNU51), and $1.0 \mathrm{~m}$ $(0.96 \mathrm{~m})$ at Kouzushima (sample K114). Since the ${ }^{14} \mathrm{C}$ content of many Group A specimens has been influenced by the atomic bomb effect, the uplift of Group A must have occurred after 1950. As noted above, Niijima and Kouzushima experienced up to 0.2 and $0.9 \mathrm{~m}$ of gradual uplift during the periods 2000-2014 and 1990-2014, 


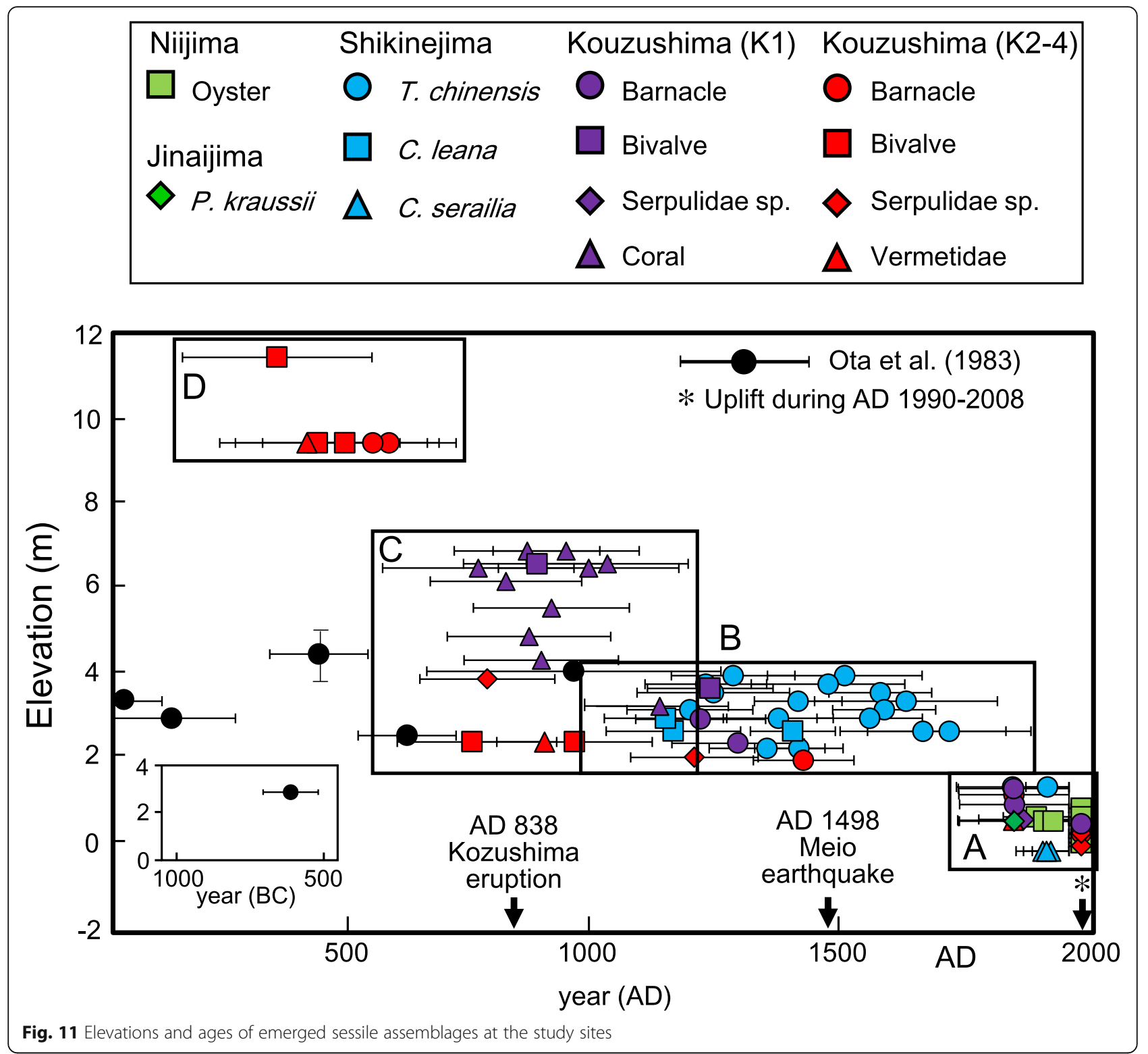

respectively (Fig. 4). These uplift events were caused by the development of a pressure source beneath Kouzushima and dike intrusion associated with the AD 2000 eruption of Miyakejima Volcano, respectively (Kimata et al. 2000; Murakami et al. 2001; Nishimura et al. 2001; Yamaoka et al. 2005). Thus, we conclude that this episode of crustal deformation, from 1990 to 2014, contributed to the emergence of Group A. The differences between our uplift estimates and values derived from instrumental data are $0.3 \mathrm{~m}$ at Niijima, $0.2 \mathrm{~m}$ at Jinaijima, and $0.4 \mathrm{~m}$ at Shikinejima, all of which can be explained by uplift during the period before GPS observation. Uplift was recorded by GPS at these sites as well as at Kouzushima. Thus, Niijima, Jinaijima, and Shikinejima may have experienced gradual uplift before GPS observation. Data of the present data were used to determine the minimum uplift that occurred at Niijima $(0.5 \mathrm{~m})$, Jinaijima (0.4 m), Shikinejima $(0.6 \mathrm{~m})$, and Kouzushima $(0.9 \mathrm{~m})$ during uplift event 1. Many specimens of C. gigas and $S$. kegaki at Niijima that were found within their living depth ranges were probably killed by burial under sand due to local changes in coastal topography.

\section{Uplift event 2}

Group B includes C. leana and T. chinensis at Shikinejima and T. chinensis, $T$. coccinea, and Serpulidae sp. at Kouzushima (Table 2). Cardita leana inhabits the middle to lower intertidal zone from -0.8 to $+0.4 \mathrm{~m}$ amsl (Okutani 2000), and T. coccinea commonly inhabits water depths of 0-60 m off southwest Japan (Sentoku 
and Ezaki 2012). Based on the vertical distribution of living species, the minimum total uplift of Group B since the time of active growth of the assemblages is estimated to be $3.3 \mathrm{~m}$ at both Shikinejima (sample ISZ61) and Kouzushima (sample K123). If the actual uplift during event 1 was equal to the minimum uplift that we estimate for event 1 in this study, then a minimum of $2.7 \mathrm{~m}$ of uplift occurred at Shikinejima and $2.4 \mathrm{~m}$ at Kouzushima during uplift event 2.

With the exception of one specimen that yields an age of AD 1539-1872 (sample TMR13), the ${ }^{14} \mathrm{C}$ age ranges of Group B specimens coincide with, or exceed, the AD 1498 Meio earthquake. The age range of sample TMR13 (AD 1539-1872) indicates that the uplift of Group B was caused by an event that occurred after the Meio earthquake. However, we do not exclude the possibility that uplift event 2 was caused by the AD 1498 Meio earthquake, because the $\sim 30$-year difference between the age of the youngest specimen and the earthquake could be explained by temporal changes in the value of the local correction to the ${ }^{14} \mathrm{C}$ age $(\Delta R)$. Hirabayashi et al. (2017) recently reported that $\Delta R$ data from Okinawa for 1900-1950 fluctuated from - $136 \pm 42$ to $62 \pm 50$ years. Since both the study area and Okinawa are in the Kuroshio region, temporal changes in $\Delta R$ will affect the calibration of ${ }^{14} \mathrm{C}$ ages used to provide calendar dates for our specimens. Thus, we cannot exclude the possibility that uplift event 2 at Shikinejima and Kouzushima was caused by the Meio earthquake, in contrast to the interpretation of Ota et al. (1983). Other possible mechanisms include another strong earthquake (i.e., $M_{\mathrm{W}} \geq 6.0$ ) or dike intrusion. Many strong earthquakes have struck the study area (Hagiwara and Omote 1936; Honda 1938; Miura 1939; Hamada 2001; Fig. 3). As noted above, dike intrusion associated with the AD 2000 eruption of Miyakejima contributed to uplift event 1 at Kouzushima, Shikinejima, and Niijima (Nishimura et al. 2001; Yamaoka et al. 2005). According to Tsukui and Suzuki (1998), Miyakejima erupted in AD 1469, which might have caused uplift event 2.

\section{Uplift event 3}

Group C is found only on Kouzushima and has a wider range of observed elevations than the other groups. This group consists of $R$. typus (site K1), T. coccinea (site K1), Chama iostoma (site K1), C. leana (site K3), V. tokyoensis (site K3), L. curta (site K3), and Serpulidae sp. (site K4) (Table 2). The bathymetric range of $V$. tokyoensis is lower intertidal $(-0.4 \mathrm{~m} \mathrm{amsl})$ to subtidal; C. iostoma is found from the lower intertidal zone to water depths of $20 \mathrm{~m}$, and L. curta inhabits the intertidal zone to depths of $20 \mathrm{~m}$ (Okutani 2000). We observed Serpulidae sp. within the intertidal zone at Kouzushima. Ogawa et al. (1996) describe Rhizotrochus niinoi, a junior synonym of $R$. typus (Cairns,
1994), as living from the intertidal zone to a maximum depth of $18 \mathrm{~m}$ in Kanaya, Chiba, Central Japan. Based on the vertical distribution of living species, the minimum total uplift of Group C since active growth ceased is estimated to be $6.9 \mathrm{~m}$ at site $\mathrm{K} 1$ (K142), $2.9 \mathrm{~m}$ at site K3 (K322), and $3.1 \mathrm{~m}$ at $\mathrm{K} 4 \mathrm{~K} 422)$. If the uplifts in events $1(0.9 \mathrm{~m})$ and 2 ( $2.4 \mathrm{~m}$ at $\mathrm{K} 1$ ) were no more than the minimum estimates, then the total uplift is $3.3 \mathrm{~m}$. Thus, we estimate that a minimum of $3.6 \mathrm{~m}$ uplift occurred at site $\mathrm{K} 1$. While the minimum total uplift of Group C at sites K3 (2.9 m) and K4 $(3.1 \mathrm{~m})$ is smaller than the total of $3.3 \mathrm{~m}$ when assumption (2) holds, the amount of uplift during events 2 and 3 at sites K3 and K4 is smaller than that at site K1. A similar spatial gradient in uplift was reported by Kimata et al. (2000), who stated that the uplift rate in the northern part of their study area was nearly twice as high as that in the southern part during 1997-2000.

All ${ }^{14} \mathrm{C}$ ages of Group $\mathrm{C}$ specimens fall within the period AD 550-1170 BC. During this interval, the AD 838 eruption of Tenjosan volcano on Kouzushima represents the most probable cause of uplift event 3. With the exception of one specimen that yields an age of AD 843-1173 (sample K1441), the ${ }^{14} \mathrm{C}$ age ranges of Group $\mathrm{C}$ specimens overlap the eruption date. The $\sim 30$-year difference between the age of the youngest specimen and the eruption could be explained by temporal changes in $\Delta R$ (Hirabayashi et al. 2017). Uplift event 3 could be explained by lava dome formation during the eruption of Tenjosan volcano (Isshiki 1982); other possible mechanisms include strong earthquakes (i.e., $M_{\mathrm{W}} \geq 6.0$ ) and dike intrusion. The AD 838886 eruption of Miyakejima (Tsukui and Suzuki 1998) may have caused uplift event 3 .

\section{Uplift event 4}

Group D, which is found only at site K2 in Kouzushima, includes Tetraclita sp., $V$. tokyoensis, C. leana, and L. curta (Table 2). Based on the vertical distribution of living species, the minimum total uplift of Group D since the time of active growth is estimated to be $11.0 \mathrm{~m}$ (sample K22). Since the other groups are not seen at site $\mathrm{K} 2$, the total amount of uplift over events 1-3, which ranges from 2.9 m (site K3) to $7.7 \mathrm{~m}$ (at site $\mathrm{K} 1$ ), indicates $3.3-8.1 \mathrm{~m}$ of uplift during event 4 .

The ${ }^{14} \mathrm{C}$ ages of Group D specimens lie in the range AD 161-686, indicating that the timing of event 4 overlaps volcanic activity that occurred about 230 years before the AD 838 eruption of Kouzushima (Togashi 1984). Thus, we propose that uplift event 4 was related to this volcanic activity, as well as strong earthquakes (i.e., $M_{\mathrm{W}} \geq 6.0$ ) and dike intrusion.

At Shikinejima, the non-calibrated ${ }^{14} \mathrm{C}$ ages of $T$. chinensis, Tetraclita japonica, Megabalanus rosa, and Saccostrea kegaki (Table 1) presented by Ota et al. (1983) are older than those obtained in this study (Fig. 11). Four specimens 
(GaK-9717, GaK-9718, GaK-9720, and GaK-9721) have elevations similar to those of Group B, so it is likely that their ${ }^{14} \mathrm{C}$ ages were influenced by contamination by dead carbon after being uplifted with Group B. Spurgeon et al. (2003) obtained radiocarbon dates of shells and cements of carbonate-cemented shell debris located along the coast of the Florida Peninsula, USA. Their results show that the cement ages were 1400-1600 years older than those of the shells. Spurgeon et al. (2003) proposed that dead carbon was introduced during the dissolution-cementation process, causing the cement to yield dates much older than when cementation likely occurred. On the other hand, the elevations of two specimens (GaK-9719 and GaK-9722) are $\sim 1.1 \mathrm{~m}$ higher than in Group B. This may indicate that uplift took place before uplift event 2 . However, no further comparison between the results of Ota et al. (1983) and this study is possible, because we cannot measure the ${ }^{14} \mathrm{C}$ ages of the specimens examined by Ota et al. (1983).

\section{Conclusions}

We re-examined Holocene crustal movements at Niijima, Jinaijima, Shikinejima, and Kouzushima based on marine species compositions and determined the AMS ${ }^{14} \mathrm{C}$ ages of emerged sessile assemblages. Our conclusions are as follows:

1. We identified four groups of emerged sessile assemblages that we infer to have been uplifted during four rapid events (uplift events 1-4). The ages of these events are estimated to be after AD 1950, AD 786-1891, AD 600-1165, and AD 161686 , respectively. The minimum amounts of uplift during events $1-4$ are estimated to be $0.4-0.9,2.4-$ 2.6, 3.6, and 3.3-8.1 $\mathrm{m}$, respectively.

2. Uplift event 1 was caused by the appearance of a pressure source and dike intrusion associated with the AD 2000 eruption of Miyakejima Volcano. These mechanisms, along with strong earthquakes, are the most likely causes of uplift events 2,3 , and 4 . Considering the timing of uplift event 2 , there is the possibility that the AD 1498 Meio earthquake caused the event. The volcanic eruption of AD 838 and a previous eruptive episode in Kouzushima are also candidate mechanisms for uplift events 3 and 4 , respectively.

\section{Abbreviations}

AMS: Accelerator mass spectrometry; amsl: Above mean sea level

\section{Acknowledgements}

We thank Drs. Toshiyuki Yamaguchi, Kaoru Sugihara, and Asuka Sentoku for assistance with identifying barnacles and corals, respectively. We thank three anonymous reviewers and associate editor Ken Ikehara for their thoughtful input into this manuscript. The sampling of specimens was conducted with a permit issued by the Agency for Cultural Affairs, Kanto Regional Ministry of the Environment.

\section{Authors' contributions}

AK conceived and designed the study and collected samples. TI identified specimens of mollusks and undertook ${ }^{14} \mathrm{C}$ analysis of the samples. TI and YM discussed the sources of crustal deformation. Ml investigated the distribution of living corals. YM and YY undertook ${ }^{14} \mathrm{C}$ analysis of the samples. YT identified specimens of corals. All authors read and approved the final manuscript.

\section{Funding}

This study was funded by Grants-in-Aid (26287126, 17H02972) awarded by the Japan Society for the Promotion of Science.

\section{Competing interests}

The authors declare that they have no competing interests.

\section{Publisher's Note}

Springer Nature remains neutral with regard to jurisdictional claims in published maps and institutional affiliations.

\section{Author details}

${ }^{1}$ Institute of Geosciences, Shizuoka University, 836 Oya, Suruga-ku, Shizuoka 422-8529, Japan. ${ }^{2}$ Center for Integrated Research and Education of Natural Hazards, Shizuoka University, 836 Oya, Suruga-ku, Shizuoka 422-8529, Japan. ${ }^{3}$ Atmosphere and Ocean Research Institute, University of Tokyo, Chiba 277-8564, Japan. ${ }^{4}$ Tottori University of Environmental Studies, 1-1 Wakabadai-kita, Tottori 689-1111, Japan.

Received: 28 December 2016 Accepted: 3 October 2017 Published online: 18 October 2017

\section{References}

Aida I (1981) Numerical experiments of historical tsunamis generated off the coast of the Tokaido district. Bull Earthquake Res Inst 56:367-390 (in Japanese with English abstract)

Ando M (1975) Source mechanisms and tectonic significance of historical earthquakes along the Nankai trough, Japan. Tectonophysics 27:119-140

Bronk Ramsey C (1995) Radiocarbon calibration and analysis of stratigraphy: the OxCal program. Radiocarbon 37(2):425-530

Bronk Ramsey C, Lee C (2013) Recent and planned development of the program OxCal. Radiocarbon 55(2-3):720-730

Cairns SD (1994) Scleractinia of the temperate North Pacific. Smithsonian Contrib Zool 557:1-150

Chamot-Rooke N, Le Pichon X (1989) Zenisu Ridge: mechanical model of formation. Tectonophysics 160(1):175-193

Coastal Movements Data Center (accessed on 3 December 2016) Table of annual mean sea level along the Japanese coast. http://cais.gsi.go.jp/cmdc/center/ annual.html\#9

Fukutomi K (1935) Crustal movements of Izu Peninsula, Central Japan, based on historical documents and oral traditions (part 1). Zisin (J Seis Soc Japan) 7: 145-153 (in Japanese)

Fukutomi K (1938) Traces of uplifts at Niijima and Shikinejima, Japan. Zisin (J Seis Soc Japan) 10:1-4 (in Japanese)

Hagiwara T, Omote S (1936) The Niizima earthquake of December 27, 1936. Bull Earthquake Res Inst 15:559-568 (in Japanese with English abstract)

Hamada M, HiramatsuY OM, Yamaguchi H (2016) Fossil tubeworms link coastal uplift of the northern Noto peninsula to rupture of the Wajima-oki fault in AD 1729. Tectonophysics 670:38-47

Hamada N (2001) A history of seismic activity around Miyakejima, Kouzushima and Niijima, the Northern Izu Islands. J Geogr 110:132-144 (in Japanese with English abstract)

Hatori T (1975) Sources of large tsunamis generated in the Boso, Tokai and Nankai regions in 1498 and 1605. Bull Earthquake Res Inst 50:171-185 (in Japanese with English abstract)

Hirabayashi S, Yokoyama Y, Suzuki A, Miyairi Y, Aze T (2017) Short-term fluctuations in regional radiocarbon reservoir age recorded in coral skeletons from the Ryukyu Islands in the north-western Pacific. J Quat Sci 32:1-6

Honda K (1938) Report of Izu-Niijima area affected by strong earthquake at 1936, 27 December. Quart J Seis 10:147-150 (In Japanese) 
Iryu Y, Maemoku H, Yamada T, Maeda Y (2009) Limestones as a paleobathymeter for reconstructing past seismic activities: Muroto-misaki, Shikoku, southwestern Japan. Glob Planet Chang 66:52-64

Ishibashi K, Ota Y, Matsuda T (1979) Radiocarbon ages of raised shell beds at the southern tip of Izu Peninsula, central Japan. Zisin (J Seismol Soc Jpn) Ser 2 32:105-107 (in Japanese)

Ishibashi K, Satake K (1998) Problems of forecasting great earthquakes in the subduction zones around Japan by means of paleoseismology. Zisin ( $J$ Seismol Soc Jpn) Ser 2 50:1-21 (in Japanese with English Abstract)

Ishihara T (1989) Gravimetric determination of the density of the Zenisu Ridge. Tectonophysics 160(1):195-205

Isshiki N (1982) Geology of the Kouzushima district. quadrangle series scale 1: 50,000. Geol Surv Jpn :34 (in Japanese with English abstract)

Isshiki N (1987) Geology of the Niijima district. quadrangle series scale 1:50,000. Geol Surv Jpn :85 (in Japanese with English abstract)

Kayanne H, Yamamuro M, Matsumoto E (1987) Pomatoleios kraussi (BAIRD) as a paleo sea level indicator on the southeast coast of Boso Peninsula, central Japan. Quat Res (Daiyonki-Kenkyu) 26:47-57 (in Japanese with English abstract)

Kimata F, Kariya S, Fujita M, Matsumoto K, Tabei T, Segawa J, Yamada A (2000) Estimated pressure source in Kozu Island volcano, southern Central Japan, with GPS measurements (July 1996-August 1999). Earth Planets Space 52:975-978

Kitamura A, Imai T, Miyairi Y, Yokoyama Y, Iryu Y. (in press) Radiocarbon dating of coastal boulders from Kouzushima and Miyakejima Islands off Tokyo metropolitan area, Japan: implications for coastal hazard risk. Quat Int. https://doi.org/10.1016/j.quaint.2017.05.040

Kitamura A, Koyama M, Itasaka K, Miyairi Y, Mori H (2014) Abrupt late Holocene uplifts of the southern Izu Peninsula, central Japan: evidence from emerged marine sessile assemblages. Island Arc 23:51-61

Kitamura A, Mitsui Y, Kawate S, Kim HY (2015) Examination of an active submarine fault off the southeast Izu Peninsula, central Japan, using field evidence for coseismic uplift and a characteristic earthquake model. Earth Planets Space 67:197. https://doi.org/10.1186/s40623-015-0367-Z

Kitamura A, Ohashi Y, Ishibashi H, Miyairi Y, Yokoyama Y, Ikuta R, Ito Y, Ikeda M. Shimano T (2016) Holocene geohazard events on the southern Izu Peninsula, central Japan. Quat Int 397:541-555

Laborel J, Laborel-Deguen F (1994) Biological indicators of relative sea-level variations and of coseismic displacements in the Mediterranean region. J Coast Res 10(2): 395-415

Lallemant S, Chamot-Rooke N, Le Pichon X, Rangin C (1989) Zenisu Ridge: a deep intraoceanic thrust related to subduction, off southwest Japan. Tectonophysics 160(1):151-174

Le Pichon X, liyama T, Boulègue J, Charvet J, Faure M, Kano K, Lallemant S, Okada H, Rangin C, Taira A, Uyeda S, Urabe T (1987) Nankai Trough and Zenisu Ridge: a deep-sea submersible survey. Earth Planet Sci Lett 83(1-4):285-299

Maemoku H (1988) Holocene crustal movement in Muroto Peninsula, southwest Japan. Geogr Rev Jpn 61:747-769 (in Japanese with English abstract)

Mazzotti S, Henry P, Le Pichon X (2001) Transient and permanent deformation of central Japan estimated by GPS: 2. strain partitioning and arc-arc collision. Earth Planet Sci Lett 184(2):455-469

Mazzotti S, Henry P, Le Pichon X, Sagiya T (1999) Strain partitioning in the zone of transition from Nankai subduction to Izu-Bonin collision (Central Japan): implications for an extensional tear within the subducting slab. Earth Planet Sci Lett 172(1):1-10

Miura T (1939) Preliminary report of focal mechanism of earthquakes at 1938, 27 December in Niijima, Japan. Quart J Seis 10(1):65-77 (in Japanese)

Miyauchi T (1990) Holocene coastal seismotectonics in the eastern margin of the sea of Japan. J Geogr (Chigaku Zasshi) 99:390-391 (in Japanese)

Murakami M, Nishimura T, Ozawa S (2001) Crustal deformation associated with the 2000 eruption of Miyake volcano and earthquake swarm near Kozu island. Bull Geospat Inf Auth Jpn 95:115--120 (in Japanese)

Nakagawa H, Toyofuku T, Kotani K, Miyahara B, Iwashita C, Kawamoto S, Hatanaka Y, Munekane H, Ishimoto M, Yutsudo T, Ishikura N, Sugawara Y (2009) Development and validation of GEONET new analysis strategy (version 4). J Geogr Surv Inst 118:1-8 (in Japanese)

Nakanishi A, Shiobara H, Hino R, Kodaira S, Kanazawa T, Shimamura H (1994) Detailed Subduction structure of the Philippine Sea plate off Tokai District deduced by Airgun-Ocean bottom seismograph profiling: crustal structure of the Zenisu Ridge and the eastern Nankai trough. Zisin (J Seismol Soc Jpn) Ser 2 47(3):311-331

Nishimura T, Ozawa S, Murakami M, Sagiya T, Tada T, Kaidzu M, Ukawa M (2001) Crustal deformation caused by magma migration in the northern Izu Islands, Japan. Geophys Res Lett 28:3745-3748
Nishimura T, Sagiya T, Stein RS (2007) Crustal block kinematics and seismic potential of the northernmost Philippine Sea plate and Izu microplate, central Japan, inferred from GPS and leveling data. J Geophys Res Solid Earth 112(B05414)

Ogawa K, Takahashi K, Tachikawa H, Chiba J (1996) A revision of the Japanese ahermatypic corals around the coastal region with guide to identification, III. Genera Rhizotrochus, Javania, Desmophyllum, Culicia, Phyllangia, and Oulangia. Nankiseibutu (Nanki Biol Soc) 38(1):37-48 (In Japanese)

Okutani T (2000) Marine mollusks in Japan. Tokai University Press, Tokyo, p 1173

Ota Y, Ishibashi K, Matsushima Y, Matsuda T, Miyoshi M, Kashima K, Matsubara A (1986) Holocene relative sea-level change in the southern part of Izu Peninsula, central Japan; data from subsurface investigation. Quat Res (Daiyonki-Kenkyu) 25:203-223 (in Japanese with English abstract)

Ota Y, Ishibashi K, Moriwaki H (1983) Late Holocene uplift of Shikine Island on the northern tip of the Philippine Sea Plate. Zisin (J Seismol Soc Jpn) Ser 2 36:587-595 (in Japanese with English abstract)

Pirazzoli PA, Stiros SC, Arnold M, Laborel J, Laborel-Deguen F (1999) Late Holocene coseismic vertical displacements and tsunami deposits near Kynos, Gulf of Euboea, Central Greece. Phys Chem Earth A 24(4):361-367

Reimer PJ, Bard E, Bayliss A, Beck JW, Blackwell PG, Bronk RC, Buck CE, Cheng H, Edwards RL, Friedrich M, Grootes PM, Guilderson TP, Haflidason H, Hajdas I, Hatté C, Heaton TJ, Hoffmann DL, Hogg AG, Hughen KA, Kaiser KF, Kromer B, Manning SW, Niu M, Reimer RW, Richards DA, Scott EM, Southon JR, Staff RA, Turney CSM, Van der Plicht J (2013) IntCal13 and Marine13 radiocarbon age calibration curves 0-50,000 years cal BP. Radiocarbon 55(4):1869-1887

Sagiya T (1999) Interplate coupling in the Tokai district, central Japan, deduced from continuous GPS data. Geophys Res Lett 26(15):2315-2318

Sato H (2008) Reconstruction of Holocene sea-level change along the coast of Harimanada in the eastern part of the Seto Inland Sea, western Japan. Quat Res (Daiyonki Kenkyu) 47:247-259 (in Japanese with English abstract)

Sayani HR, Cobb KM, Cohen AL, Elliott WC, Nurhati IS, Dunbar RB, Rose KA, Zaunbrecher LK (2011) Effects of diagenesis on paleoclimate reconstructions from modern and young fossil corals. Geochim Cosmochim Acta 75:6361-6373

Sentoku A, Ezaki Y (2012) Regularity in budding mode and resultant growth morphology of the azooxanthellate colonial scleractinian Tubastraea coccinea. Coral Reefs 31:67-74

Shishikura M (2014) History of the paleo-earthquakes along the Sagami trough, central Japan: review of coastal paleoseismological studies in the Kanto region. Episodes 37:246-257

Spurgeon D, Davis RA Jr, Shinnu EA (2003) Formation of 'beach rock' at Siesta Key, Florida and its influence on barrier island development. Mar Geol 200:19-29

Tanabe S, Hori K, Momohara A, Nakashima R (2016) Verification of the "Yayoi regression" in the Tonegawa Lowland, central Japan. J Geol Soc Jpn 122: 135-153 (in Japanese with English abstract)

Tanigawa K, Hyodo M, Sato H (2013) Holocene relative sea-level changes and rate of sea-level rise from coastal deposits in the Toyooka Basin, western Japan. The Holocene 23:1039-1051

Togashi S (1984) ${ }^{14} \mathrm{C}$ ages of charcoal from pyroclastics of Tenjosan volcano, Kouzushima, the Izu Islands, Japan. Volcanol Soc Jpn II 4:277-283

Tsukui M, Suzuki Y (1998) Eruptive history of Miyakejima volcano during the last 7000 years. Volcanol Soc Jpn II 43:149-166

Woodroffe CD, McGregor HV, Lambeck K, Smithers SG, Fink D (2012) Mid-Pacific microatolls record sea-level stability over the past 5000 yr. Geology 40(10): 951-954

Yamaguchi T, Hisatsune Y (2006) Taxonomy and identification of Japanese barnacles. Sessile Org 23(1):1-15

Yamaoka K, Kawamura M, Kimata F, Fujii N, Kudo T (2005) Dike intrusion associated with the 2000 eruption of Miyakejima volcano, Japan. Bull Volcanol 67:231-242

Yokoyama Y, Anderson J, Yamane M, Simkins LM, Miyairi Y, Yamazaki T, Koizumi M, Suga H, Kusahara K, Prothro L, Hasumi H, Southon JR, Ohkouchi N (2016) Widespread collapse of the Ross ice shelf during the late Holocene. Proc Natl Acad Sci U S A 113(9):2354-2359

Yokoyama Y, Okuno J, Miyairi Y, Obrochta SP, Demboya N, Makino Y, Kawahata H (2012) Holocene sea-level change and Antarctic melting history derived from geological observations and geophysical modeling along the Shimokita peninsula, northern Japan. Geophys Res Lett 39:L13502. https://doi.org/10. 1029/2012GL051983

Yoneda M, Kitagawa H, van der Plicht J, Ushida M, Tanaka A, Uehiro T, Shibata Y, Morita M, Ohno T (2000) Pre-bomb marine reservoir ages in the western north Pacific: preliminary result on Kyoto University collection. Nucl Instrum Methods Phys Res Sect B 172(1):377-381 